\title{
Unified Framework for the Synchronization of Flexible Multicarrier Communication Signals
}

\author{
José A. López-Salcedo, Member, IEEE, Esteban Gutiérrez, Gonzalo Seco-Granados, Senior Member, IEEE, and \\ A. Lee Swindlehurst, Fellow, IEEE
}

\begin{abstract}
This paper presents a unified framework for the formulation of synchronization algorithms dealing with the general class of Filter-Bank Multi-Carrier (FBMC) communication signals. This is a wide family of signaling formats that includes Orthogonal Frequency Division Multiplexing (OFDM), for instance, as just one of many particular cases. One of the main contributions of this work is the proposal of a novel matrix signal model for so-called flexible FBMC signals, in which no restrictions are imposed on the signal design parameters (i.e., pulse shaping, symbol rate, carrier spacing, sampling frequency, etc.), unlike the type of multicarrier signals currently deployed. As an example of application of the proposed matrix signal model, blind joint timedelay and frequency estimators will be derived under both the Conditional Maximum Likelihood (CML) and the low-SNR Unconditional Maximum Likelihood (UML) principles, for any FBMC signal propagating through an arbitrary multipath channel. These estimators will be specialized first for the case of critically sampled cyclic-prefix OFDM (CP-OFDM) signals in frequency flat fading, leading to simple architectures amenable to a hardware implementation. Later on, and for the low-SNR UML principle, the special case of critically sampled CP-OFDM in multipath channels will be addressed, where a novel synchronizer will be proposed.
\end{abstract}

Index Terms-Carrier frequency estimation, conditional maximum likelihood (CML), filterbank multicarrier (FBMC), multicarrier signals, multipath, orthogonal frequency division multiplexing (OFDM), time-delay estimation, unconditional maximum likelihood (UML).

\section{INTRODUCTION}

$\mathbf{M}$ ULTICARRIER (MC) signals are currently the preferred modulation scheme in a myriad of different communication systems such as wide-area networks (LTE, WiMAX), local-area networks (IEEE $802.11 \mathrm{~g}$ ), broadcasting

Manuscript received April 23, 2012; revised August 24, 2012; accepted October 14, 2012. Date of publication November 27, 2012; date of current version January 23,2013 . The associate editor coordinating the review of this manuscript and approving it for publication was Prof. Sofiene Affes. This work was supported by the Spanish Government project TEC2011-28219 and by the 2010-2011 California-Catalonia Engineering Innovation Program funded by the Balsells-Generalitat Fellowship.

J. A. López-Salcedo and G. Seco-Granados are with the Department of Telecommunications and Systems Engineering, Universitat Autònoma de Barcelona, 08193 Bellaterra, Barcelona, Spain (e-mail: jose.salcedo@uab.es; gonzalo.seco@uab.es).

E. Gutiérrez is with the Aerospace Research and Technology Centre (Ascamm Foundation), 08290 Cerdanyola del Vallés, Spain (e-mail: egutierrez@ascamm.com).

A. L. Swindlehurst is with the Department of Electrical Engineering and Computer Science, University of California at Irvine, Irvine, CA 92697 USA (e-mail: swindle@uci.edu).

Color versions of one or more of the figures in this paper are available online at http://ieeexplore.ieee.org.

Digital Object Identifier 10.1109/TSP.2012.2229997
(DVB-T/H, DRM, DAB, DMB) and short-range communication (UWB) [1]. The reasons for such a wide consensus are the indisputable advantages of MC signals when compared to other modulation schemes. For instance, MC signals make it possible to convert a time dispersive channel into a multiplicative one, thus significantly reducing the equalization complexity at the receiver side. High spectral efficiency can also be achieved by dividing the available transmission bandwidth into several (even thousands) of narrowband subchannels, where power can be allocated in a dynamic way. This leads to the so-called water-filling schemes, successfully adopted in wired xDSL systems for maximizing the channel capacity of last mile communication links [2]. Moreover, such an efficient subdivision of the spectrum can also be used for implementing media access control (MAC) based on agile frequency division [3], as currently being considered in the LTE downlink or in opportunistic wireless networks.

Nevertheless, these advantages are as numerous and diverse as the different possible versions and implementations of MC signals. Orthogonal Frequency Division Multiplexing (OFDM) is certainly the most popular one, but Offset Quadrature Amplitude Modulated OFDM (OQAM/OFDM), Discrete Multitone (DMT), Filtered Multitone Modulation (FMT), Discrete Wavelet Multi-Tone (DWMT) or Filter-Bank Multi-Carrier (FBMC) can also be found in many practical applications. The latter, indeed, has often been reported as a better solution than traditional OFDM in multiple access [4] and cognitive radio applications [5]. The problem, however, is that each particular variation of MC signal has received an individual treatment in the literature. This involves using a very specific signal model and design assumptions, thus making it very difficult to extrapolate the results to other types of MC signals.

In this context, the purpose of this paper is to introduce a unifying framework to represent, analyze and assess the performance of the general class of MC signals. To do so, we focus on so-called flexible FBMC signals, from which any of the above mentioned $\mathrm{MC}$ variations can be obtained by varying the pulse shape filter, the subcarrier spacing, the MC symbol duration or the subcarrier period [6]. This is in contrast with traditional OFDM signaling, for instance, where a rectangular pulse shape is adopted and the subcarrier separation is forced to be equal to the inverse of the effective MC symbol period. Another advantage of our proposed flexible FBMC signal model is its novel matrix notation, as opposed to the scalar signal model typically adopted in the MC literature. Matrix notation provides an insightful interpretation of the operations to be carried out at the receiver, and interestingly, it also allows us to exploit many of the existing results in the field of array signal processing, where matrix algebra has been widely and successfully adopted. 
As an example of the applicability of the proposed matrix signal model, the present contribution concentrates on the optimal blind synchronization problem, in which the MC symbol time-delay and the carrier frequency offset are estimated without the benefit of pilot symbols. The problem of blind synchronization has received significant attention in recent years as an alternative to pilot-based synchronization [7], which suffers from a significant penalty in terms of effective throughput. Among the several contributions in this field, [8] was one of the first to address this problem in an optimal manner by using the Maximum Likelihood (ML) principle. The solution proposed in [8] and later on in [9] was derived for critically sampled ${ }^{1}$ OFDM signals assumed to be Gaussian distributed, which only applies when the number of subcarriers is asymptotically large [10]. A cyclic prefix (CP) was also assumed to be appended at the beginning of each OFDM symbol, but since the channel was initially assumed to be frequency flat in [8], [9], the actual role of the cyclic prefix (consciously or not) was to serve for synchronization purposes rather than for avoiding intersymbol interference caused by dispersive channels. Indeed, the resulting synchronizer was found to rely on the correlation between the received signal and a time-shifted version of itself, where the $\mathrm{CP}$ samples of one replica matched the original samples of the other. The need for a CP in critically sampled OFDM was already highlighted in [11], where it was shown that blind synchronization is not possible unless some kind of redundancy is incorporated. Such a redundancy allows critically sampled OFDM signals to exhibit second-order cyclostationarity, thus enabling blind synchronization [12]. This observation has led many contributions to bypass the optimal formulation of OFDM blind synchronization problems, and to focus instead on exploiting the second-order statistics of the received signal in a straigthforward manner. This is the case in [11] and also in [13]-[15].

The present contribution departs from previous approaches in the literature by making use of our unified matrix signal model, and thus it addresses the general problem of Maximum Likelihood (ML) synchronization for flexible FBMC signals. Both the Conditional Maximum Likelihood (CML) and the low-SNR Unconditional Maximum Likelihood (UML) estimators will derived for any FBMC signal propagating through an arbitrary multipath channel. These results will serve as a starting point for specialized studies involving particular choices of the FBMC parameters and propagation model. As an example of one such special case, we will concentrate first on single-path (i.e., frequency flat) channels and critically sampled CP-OFDM signals. While flat fading is a strongly limiting assumption, it is interesting to be studied because the general FBMC framework leads to a simple and direct architecture that is amenable to a hardware implementation. Later on, and for the low-SNR UML principle, we will extend the results to the special case of critically sampled CP-OFDM signals propagating through multipath channels, where a novel closed-form synchronizer will be proposed.

The paper is structured as follows: the unified signal model for flexible FBMC signals will be introduced in Section II, first in its scalar form, and second in its matrix form. Based on this general signal model, the ML synchronization problem is addressed in Section III, where both CML and low-SNR UML

\footnotetext{
1"Critically sampled" means the number of samples per OFDM symbol (after cyclic prefix removal) coincides with the number of subcarriers.
}

are applied to the general class of flexible FBMC signals. The special case of CP-OFDM is presented in the next sections, with Section IV covering the CML approach for single-path channels and Section V the low-SNR UML approach for both single-path and multipath channels. Finally, simulation results are shown in Section VI and conclusions are drawn in Section VII.

\section{Unified Signal Model for Flexible FBMC Signals}

\section{A. Scalar Signal Model for the AWGN Channel}

For the sake of simplicity, let us first consider the case of an ideal Additive White Gaussian Noise (AWGN) channel. Then, a flexible FBMC signal formed by $N$ subcarriers with a frequency separation of $F_{0} \doteq 1 / T_{0}$ will be assumed, with the following continuous-time baseband received signal model:

$$
\begin{aligned}
r_{c}(t)=\sum_{l=-\infty}^{\infty} \sum_{n=0}^{N-1} & x_{n}^{\prime}(l) g_{c}\left(t-l T-t_{\epsilon}\right) \\
& \times e^{j\left(2 \pi n \frac{t-t_{\epsilon}}{T_{0}}+2 \pi f_{\epsilon} t+\theta_{\epsilon}+\varphi_{n}(l)\right)}+w_{c}(t)
\end{aligned}
$$

where $x_{n}^{\prime}(l)$ are the complex-valued symbols transmitted at the $n$-th subcarrier and $l$-th multicarrier (MC) symbol, $g_{c}(t)$ is the continuous-time shaping waveform, $T$ is the MC symbol period with $R \doteq 1 / T$ the $\mathrm{MC}$ signaling rate, and $w_{c}(t)$ is continuous-time white Gaussian noise. Note that if a cyclic prefix is added, the signal model in (1) is still valid by incorporating some appropriate phase rotation $\varphi_{n}(l)$ to each of the transmitted symbols $x_{n}(l)$. To simplify the notation, we can gather the symbols and the additional phases into an equivalent symbol term $x_{n}(l) \doteq x_{n}^{\prime}(l) e^{j \varphi_{n}(l)}$. Next, and because of the propagation delay and mismatches between transmit and receive oscillators, the received signal is usually affected by synchronization errors, namely, the continuous-time symbol timing error $t_{\epsilon}$, carrier-frequency error $f_{\epsilon}$ and carrier phase error $\theta_{\epsilon}$. At the receiver, the continuous-time signal in (1) is passed through an anti-aliasing filter and sampled at a rate of $F_{s}=1 / T_{s}$ samples per second, leading to the following discrete-time signal model:

$$
\begin{aligned}
r(m) \doteq & r_{c}\left(m T_{s}\right) \\
= & \sum_{l=-\infty}^{\infty} \sum_{n=0}^{N-1} x_{n}(l) g\left(m-l N_{s s}-\tau\right) \\
& \times e^{j\left((2 \pi / P)(n+\nu) m+\theta_{\epsilon}-(2 \pi / P) n \tau\right)}+w(m)
\end{aligned}
$$

where the fundamental subcarrier discrete-time period (expressed in samples) is $P \doteq F_{s} / F_{0}$ and the number of samples per MC symbol is $N_{s s} \doteq F_{s} T=F_{s} / R$. Note that this is the most general possible signal model, since no restrictions are imposed on either $P, N$ or $N_{s s}$, except that they are assumed to be positive integers. The discrete-time shaping pulse $g(m) \doteq g_{c}\left(m T_{s}\right)$ has a total length of $L_{g}$ samples and the white Gaussian noise samples $w(m) \doteq w_{c}\left(m T_{s}\right)$ are statistically uncorrelated with $w(m) \sim \mathcal{N}\left(0, \sigma_{w}^{2}\right)$. Regarding the synchronization parameters, the discrete-time symbol-timing error $\tau \doteq t_{\epsilon} / T_{s}$ is constrained within the interval $\left[-N_{s s} / 2,+N_{s s} / 2\right)$ and the discrete-time carrier frequency error has been normalized to the subcarrier spacing, so that $\nu \doteq f_{\epsilon} / F_{0}$ and thus $\nu \in[-P / 2,+P / 2)$. 
TABLE I

Specialization of the Unified Signal Model in (2) to the Most Popular MC Signals

\begin{tabular}{|c|c|c|c|c|c|c|}
\hline MC modulation & $\begin{array}{l}\text { Symbol } \\
\text { period }\end{array}$ & $\begin{array}{l}\text { Subcarrier } \\
\text { period }\end{array}$ & $\begin{array}{c}\text { Transmitted } \\
\text { symbols } \\
x_{n}^{\prime}(l) \\
\end{array}$ & $\begin{array}{l}\text { Phase } \\
\text { rotation }\end{array}$ & $\begin{array}{l}\text { Pulse } \\
\text { shape }\end{array}$ & $\begin{array}{l}\text { Pulse } \\
\text { length }\end{array}$ \\
\hline FBMC & $N_{s s}$ & $P$ & $s_{n}(l)$ & $\varphi_{n}(l)$ & $g(m)$ & $\geq N_{s s}$ \\
\hline OFDM + CP & $N_{s s}$ & $N$ & $s_{n}(l)$ & $-\frac{2 \pi}{N} n N_{\mathrm{scp}}$ & 1 & $N_{s s}$ \\
\hline $\mathrm{DMT}+\mathrm{CP}$ & $N_{s s}$ & $N$ & $x_{n}^{\prime}(l)=\left[x_{N-n}^{\prime}(l)\right]^{*}$ & $-\frac{2 \pi}{N} n N_{\mathrm{scp}}$ & 1 & $N_{s s}$ \\
\hline OFDM + ZP & $N_{s s}$ & $N$ & $s_{n}(l)$ & 0 & 1 & $N$ \\
\hline OQAM/OFDM & $\frac{N}{2}$ & $N$ & $\begin{array}{l}\operatorname{Re}\left[s_{n}\left(\left\lfloor\frac{l}{2}\right\rfloor\right)\right], l \text { even } \\
j \operatorname{Im}\left[s_{n}\left(\left\lfloor\frac{l}{2}\right\rfloor\right)\right], l \text { odd }\end{array}$ & 0 & $\left.g(m)\right|_{\text {OQAM }}$ & $k_{g} N, k_{g} \in \mathbb{Z}^{+} \geq 2$ \\
\hline DWMT & $N$ & $2 N$ & $\begin{array}{l}\operatorname{Re}\left[s_{\lfloor n / 2\rfloor}(l)\right], n \text { even } \\
j \operatorname{Im}\left[s_{\lfloor n / 2\rfloor}(l)\right], n \text { odd }\end{array}$ & 0 & $\left.g(m)\right|_{\text {DWMT }}$ & $k_{g} N, k_{g} \in \mathbb{Z}^{+} \geq 2$ \\
\hline FMT & $N$ & $N$ & $s_{n}(l)$ & 0 & $\left.g(m)\right|_{\text {FMT }}$ & $k_{g} N, k_{g} \in \mathbb{Z}^{+} \geq 2$ \\
\hline
\end{tabular}

\section{B. Specialization to Different Multicarrier Signal Formats}

The signal model in (2) is able to represent any specific MC signaling format by just configuring a given set of parameters. An example is shown in Table I for the most popular MC formats typically found in practice. We are assuming in Table I that a stream of complex source symbols $s_{n}(l) \in \mathbb{C}$ is transmitted using a total of $N$ subcarriers, and ${ }^{*}$ stands for the complex conjugate. Moreover, for each MC signal requiring pulse shaping, a different pulse $g(m)$ is adopted in order to reflect the freedom in selecting the pulse depending on the different constraints that may be imposed on each signaling format under consideration.

\section{Matrix Signal Model for the AWGN Channel}

In order to facilitate the mathematical treatment, the scalar discrete-time signal model in (2) is henceforth represented in matrix notation. This is a very convenient approach that allows us to gain many insights in terms of signal subspaces. Matrix data models have been used for decades in radar and array signal processing, and are progressively being adopted for the formulation of synchronization problems dealing with digital linear modulation. We will follow the same approach herein by taking into consideration a symmetric observation interval comprising $L \doteq 2 K+1$ transmitted MC symbols, and a total of $Q \doteq 2 M+1$ received samples according to the following signal model,

$$
\mathbf{r}=\mathbf{A}(\boldsymbol{\Theta}) \mathbf{x}+\mathbf{w}
$$

where $\mathbf{r} \doteq[r(-M), r(-M+1), \ldots, r(M)]^{T}$ is the $(Q \times 1)$ vector of noise-corrupted received samples, $\mathbf{w} \doteq[w(-M), w(-M+1), \ldots, w(M)]^{T}$ the $(Q \times 1)$ vector of noise samples, and $\{\mathbf{A}(\boldsymbol{\Theta}), \mathbf{x}\}$ are the shaping matrix and the vector of transmitted symbols, respectively, whose structure remains to be determined. Note that the shaping matrix explicitly depends on the vector of unknown discrete-time synchronization parameters $\Theta \doteq\left[\tau, \nu, \theta_{\epsilon}\right]^{T}$. Moreover, the linear matrix model in (3) is nothing but the statement that the FBMC signal introduced in (2) involves a linear modulation of the transmitted symbols. In one way or another, any given linear modulation can always be expressed by the general linear model in (3). The problem at hand is to determine the structure of the shaping matrix $\mathbf{A}(\boldsymbol{\Theta})$ for flexible FBMC signals. For the well-known case of single-carrier digital modulations, $\mathbf{x}$ is a ( $L \times 1$ ) vector containing the $L$ (single-carrier) symbols to be transmitted, and $\mathbf{A}(\boldsymbol{\Theta})$ turns out to be a $(Q \times L)$ matrix whose columns are simply $N_{s s}$ time-shifted replicas of the pulse shape $g(m)$ affected by all synchronization errors [16].

For the case of FBMC signals, the structure of $\mathbf{A}(\Theta)$ is not so straightforward due to the presence of multiple data-modulated subcarriers that typically exhibit a continuous phase behavior between consecutive MC symbols. For a finite observation interval of $L \mathrm{MC}$ symbols, the FBMC received signal in (2) can be written as the aggregation of $L$ amplitude-modulated waveforms with a time-shift of $N_{s s}$ samples between successive waveforms. Each waveform, in turn, is the result of the sample-by-sample product between the pulse shape $g(m)$ and the aggregation of $N$ amplitude-modulated subcarriers. That is,

$$
\mathbf{r}=\sum_{l=-K}^{K} \mathbf{u}_{l}(\tau) \odot \mathbf{g}_{l}(\boldsymbol{\Theta})+\mathbf{w}
$$

where ' $\odot$ ' stands for the Schur-Hadamard (i.e., element-wise) product and the $(Q \times 1)$ vector $\mathbf{g}_{l}$ is such that its $m$-th entry is given by $\left[\mathbf{g}_{l}(\mathbf{\Theta})\right]_{m} \doteq g\left(m-l N_{s s}-\tau\right) e^{j\left((2 \pi / P) \nu m+\theta_{\epsilon}\right)}$. Alternatively, we could also write $\mathbf{g}_{l}(\boldsymbol{\Theta})=\mathbf{g}_{l}(\tau) \odot \mathbf{e}_{Q}\left(\nu, \theta_{\epsilon}\right)$ by separating the effect of the time-delay from the carrier related errors. The latter are included within the $(i \times 1)$ vector $\mathbf{e}_{i}\left(\nu, \theta_{\epsilon}\right)$, whose $m$-th entry is given by $\left[\mathbf{e}_{i}\left(\nu, \theta_{\epsilon}\right)\right]_{m} \doteq e^{j\left((2 \pi / P) \nu m+\theta_{\epsilon}\right)}$. The $m$-th entry of the $(Q \times 1)$ vector $\mathbf{u}_{l}$ is given by $\left[\mathbf{u}_{l}(\tau)\right]_{m} \doteq \sum_{n=0}^{N-1} x_{n}(l) e^{j(2 \pi / P) n(m-\tau)}$, which is nothing but the $m$-th output bin of the $P$-point inverse discrete Fourier transform (IDFT) of the sequence of frequency-domain symbols corresponding to the $l$-th $\mathrm{MC}$ symbol, $x_{n}[l]$ for $n=0,1, \ldots, N-1$, and shifted by the time-delay $\tau$. Due to the cyclic structure of the IDFT, $\left[\mathbf{u}_{l}\right]_{m}=\left[\mathbf{u}_{l}\right]_{\bmod (m, P)}$ where $\bmod (x, y)$ stands for $x$ modulo $y$, thus highlighting the presence of a $P$-periodic signal superimposed on the traditional $N_{s s}$ cyclo-periodicity of the pulse amplitude modulation. Such a co-existence of two different periodicities complicates the transmit and receive architectures, and for this reason it is often avoided in practice by forcing $P=N_{s s}$, as in most OFDM implementations. However, more general (i.e., flexible) schemes may not necessarily have to agree in their carrier and symbol 
periodicities, thus leading to more sophisticated architectures such as the ones discussed in [6].

Coming back to the formulation in (4), standard matrix manipulations lead to the equivalent form

$$
\begin{aligned}
\mathbf{r} & =\operatorname{diag}\left(\sum_{l=-K}^{K} \mathbf{u}_{l}(\tau) \mathbf{g}_{l}^{T}(\boldsymbol{\Theta})\right)+\mathbf{w} \\
& =\operatorname{diag}\left(\mathbf{U}_{\tau} \mathbf{G}^{T}(\boldsymbol{\Theta})\right)+\mathbf{w}
\end{aligned}
$$

where $\mathbf{G}(\boldsymbol{\Theta})$ is a $(Q \times L)$ matrix defined as $\mathbf{G}(\boldsymbol{\Theta}) \doteq$ $\left[\mathbf{g}_{-K}(\boldsymbol{\Theta}), \mathbf{g}_{-K+1}(\boldsymbol{\Theta}), \ldots, \mathbf{g}_{K}(\boldsymbol{\Theta})\right]$ and $\mathbf{U}_{\tau}$ is a $(Q \times L)$ matrix resulting from the column stacking of $\mathbf{u}_{l}$ as follows, $\mathbf{U}_{\tau} \doteq\left[\mathbf{u}_{-K}(\tau), \mathbf{u}_{-K+1}(\tau), \ldots, \mathbf{u}_{K}(\tau)\right]$. Since $\mathbf{u}_{l}(\tau)$ is related to the cyclic extension of a $P$-point IDFT, it can also be written as $\mathbf{u}_{l}(\tau)=\tilde{\mathbf{F}}_{\tau}^{H} \mathbf{x}(l)$ with $\tilde{\mathbf{F}}_{\tau}^{H} \doteq \tilde{\mathbf{F}}^{H} \operatorname{diag}\left(\mathbf{e}_{N}(-\tau, 0)\right)$ and where $\tilde{\mathbf{F}}^{H}$ is a $(Q \times N)$ matrix resulting from cyclically extending the columns of a $(P \times P)$ IDFT matrix $\mathbf{F}_{P}^{H}$ truncated to $N$ columns. Assuming without loss of generality, that $Q$ is a multiple of $P$, that is $Q=k_{0} P$, with $k_{0}$ a positive integer, then $\tilde{\mathbf{F}}_{\tau}^{H}$ can be expressed in an alternative more compact manner as:

$$
\begin{aligned}
\tilde{\mathbf{F}}_{\tau}^{H} & =\mathbf{1}_{k_{0}} \otimes\left(\mathbf{F}_{P, \tau}^{H} \mathbf{T}_{P \times N}\right) \\
\mathbf{F}_{P, \tau}^{H} & \doteq \mathbf{F}_{P}^{H} \operatorname{diag}\left(\mathbf{e}_{\mathrm{P}}(-\tau, 0)\right),
\end{aligned}
$$

with $\mathbf{1}_{k_{0}}$ a $\left(k_{0} \times 1\right)$ vector of ones, $\otimes$ the Kronecker product, and $\mathbf{T}_{P \times N}$ a $(P \times N)$ zero-padding matrix $\mathbf{T}_{P \times N}=\left[\begin{array}{ll}\mathbf{I}_{N} & \mathbf{0}_{N \times(P-N)}\end{array}\right]^{T}$. According to these observations, the signal in (6) can be expressed as,

$$
\mathbf{r}=\operatorname{diag}\left(\tilde{\mathbf{F}}_{\tau}^{H} \mathbf{X G}^{T}(\boldsymbol{\Theta})\right)+\mathbf{w}
$$

where $\mathbf{X} \doteq[\mathbf{x}(-K), \mathbf{x}(-K+1), \ldots, \mathbf{x}(K)]$ is a $(N \times L)$ matrix whose $i$-th column contains the symbols on each of the $N$ subcarriers of the $i$-th MC symbol: $\mathbf{x}(l) \doteq\left[x_{0}(l), x_{1}(1), \ldots, x_{N-1}(l)\right]^{T}$.

Despite the effort in providing a compact matrix formulation for the signal in (2), some matrix products still remain inside the diagonal operator, which further complicates the understanding and the practical computation of the received signal samples. It is at this point where a more insightful result can be obtained by applying the following lemma from [17]:

Lemma 1: The diagonal entries of any $(S \times S)$ square matrix $\mathbf{M}$ can algebraically be obtained as

$$
\operatorname{diag}(\mathbf{M})=\mathbf{J}_{1}^{T}\left(\mathbf{I}_{S} \otimes \mathbf{M}\right) \mathbf{J}_{2}
$$

where $\mathbf{I}_{S}$ is the $(S \times S)$ identity matrix, $\mathbf{J}_{1} \doteq\left(\mathbf{I}_{S} \diamond \mathbf{I}_{S}\right)$ and $\mathbf{J}_{2} \doteq \operatorname{vec}(\mathbf{I})_{S}$, with ' $\diamond$ ' the Khatri-rao product and $\operatorname{vec}(\cdot)$ the column-wise stacking or vec operator.

Applying the previous lemma to (9) with $S=Q$, and after some manipulations, we get

$$
\mathbf{r}=\mathbf{J}_{1}^{T}\left(\mathbf{G}(\boldsymbol{\Theta}) \otimes\left(\tilde{\mathbf{F}}_{\tau}^{H}\right)\right) \operatorname{vec}(\mathbf{X})+\mathbf{w}
$$

Thus, we are finally in the position to claim that the linear signal model $\mathbf{r}=\mathbf{A}(\boldsymbol{\Theta}) \mathbf{x}+\mathbf{w}$ introduced in (3) is applicable to flexible FBMC communication signals with

$$
\begin{aligned}
\mathbf{A}(\boldsymbol{\Theta}) & \doteq \mathbf{J}_{1}^{T}\left(\mathbf{G}(\boldsymbol{\Theta}) \otimes \tilde{\mathbf{F}}_{\tau}^{H}\right) \\
\mathbf{x} & \doteq \operatorname{vec}(\mathbf{X}) .
\end{aligned}
$$

A new $(N L \times 1)$ vector $\mathbf{x}$ is defined, encompassing all the timefrequency symbols to be transmitted. In practice, it may be of interest to explicitly indicate the existence of data or pilot symbols. This can easily be done by further splitting $\mathbf{x}$ as follows,

$$
\mathbf{x}=\mathbf{T}_{d} \mathbf{x}_{d}+\mathbf{T}_{p} \mathbf{x}_{p}
$$

where the reduced-length vectors $\mathbf{x}_{p}\left(N_{p} \times 1\right)$ and $\mathbf{x}_{d}\left(N_{d} \times\right.$ 1) contain the $N_{p}$ and $N_{d}$ time-frequency pilot and data symbols, respectively, with $N_{p}+N_{d}=N L$. The placement of the time-frequency pilot and data symbols is defined by the selection matrices $\mathbf{T}_{p}\left(N L \times N_{p}\right)$ and $\mathbf{T}_{d}\left(N L \times N_{d}\right)$, which contain only ones and zeros. It can also be verified that $\mathbf{T}_{p}^{H} \mathbf{T}_{p}=\mathbf{I}_{N_{p}}$, $\mathbf{T}_{d}^{H} \mathbf{T}_{d}=\mathbf{I}_{N_{d}}$ and $\mathbf{T}_{d}^{H} \mathbf{T}_{p}=\mathbf{0}$.

\section{Matrix Signal Model for Multipath Channels}

The signal model presented so far is implicitly applicable to frequency flat fading channels such as those normally experienced in fixed satellite links, where in addition to the propagation delay, just a relatively slow time-varying attenuation is incurred. However, most wireless communication devices are subject to much more challenging propagation conditions involving channels with multipath or frequency-selective fading, whose continuous-time impulse response can be expressed as

$$
h_{c}(t)=\sum_{k=0}^{L_{h}-1} h_{k} \delta\left(t-k T_{s}-t_{\epsilon}\right)
$$

with $L_{h}$ a known upper bound on the number of discrete multipath components, $h_{k}$ the complex gain for the $k$-th path, and $t_{\epsilon}$ the time-delay introduced by the channel (i.e., the time-delay of the first path). This model is typically preferred over the tappeddelay line model because it explicitly depends on a single timedelay, $t_{\epsilon}$, which is indeed the parameter of interest for synchronization or ranging purposes [18, Section III-B], [19]. In the discrete-time domain, the channel in (15) results in

$$
h(m)=\sum_{k=0}^{L_{h}-1} h_{k} \operatorname{sinc}(m-k-\tau)
$$

where $h(m)$ is restricted in practice to $Q_{h} \doteq 2 M_{h}+1$ samples at most, with $m=-M_{h}, \ldots, M_{h}$. Note also that since the multipath channel gains are complex, the constant carrier phase error $\theta_{\epsilon}$ previously defined for single-path channels now becomes superfluous, and thus will be ignored.

With these considerations in mind, the matrix signal model in Section II-C is extended to include the effect of multipath channels as follows:

$$
\mathbf{r}=\mathbf{H}_{\tau} \mathbf{A}_{\nu} \mathbf{x}+\mathbf{w}
$$

where $\mathbf{r}$ is now a $\left(Q_{r} \times 1\right)$ vector in order to encompass the $Q_{h}$-sample increase due to the propagation through the channel, with $Q_{r} \doteq Q+Q_{h}-1$. The $\left(Q_{r} \times Q\right)$ channel matrix $\mathbf{H}_{\tau}$ is defined as

$$
\mathbf{H}_{\tau} \doteq \sum_{k=0}^{L_{h}-1} h_{k} \mathbf{H}_{k, \tau}
$$


and is the superposition of $L_{h}$ Toeplitz matrices $\left\{\mathbf{H}_{k, \tau}\right\}_{k=0}^{L_{h}-1}$ whose columns are one-sample shifted replicas of a time-delayed sinc function according to (16). The incorporation of the time-delay as part of the channel leads the shaping matrix $\mathbf{A}$ to depend on the multiplicative carrier frequency error only. This will be explicitly indicated by the notation $\mathbf{A}_{\nu}$. In view of this formulation, the FBMC received signal model for the general multipath fading case is defined by

$$
\begin{aligned}
\mathbf{r} & =\mathbf{B}(\boldsymbol{\Theta}) \mathbf{x}+\mathbf{w} \\
\mathbf{B}(\boldsymbol{\Theta}) & \doteq \mathbf{H}_{\tau} \mathbf{A}_{\nu} \\
\mathbf{A}_{\nu} & \doteq \mathbf{J}_{1}^{T}\left(\mathbf{G}_{\nu} \otimes \tilde{\mathbf{F}}^{H}\right) \\
\mathbf{G}_{\nu} & \doteq \operatorname{diag}\left(\mathbf{e}_{Q}(\nu, 0)\right) \mathbf{G} .
\end{aligned}
$$

The overall vector of unknown parameters thus becomes $\Theta \doteq$ $\left[h_{0}, \ldots, h_{L_{h}-1}, \tau, \nu\right]^{T}$, which includes the complex path gains, the global time-delay and the frequency error. This signal model can also be used for the single-path channel in Section II-C by fixing $L_{h}=1$, and obtaining the constant phase error as $\theta_{\epsilon}=\measuredangle\left(h_{0}\right)$. The advantage of using the above channel-dependent formulation even for the single-path case is that it easily accommodates fractional time delays without the need to change the pulse shape waveform. This is because the time-delay is now part of the channel and not part of the pulse shape itself. This is particularly convenient when rectangular pulse shapes are adopted, as in OFDM. Thanks to this flexibility, the matrix signal model in (19)-(22) will be henceforth adopted throughout the paper. The distinction between single-path and multipath scenarios will be explicitly noted as required.

\section{MAXIMUM LIKELIHOOD SYNCHRONIZATION OF FBMC SIGNALS}

The present section aims at shedding some light on the advantages that the proposed matrix signal model can bring to the field of FBMC synchronization. Since optimal synchronizers are desired, the ML principle will be adopted, which guarantees asymptotically unbiased and consistent estimates for the parameters of interest. Unfortunately, the presence of data symbols within the transmitted signal hinders the estimation of these parameters, acting as a kind of multiplicative noise. In that sense, data symbols are considered to be nuisance parameters for the estimation problem at hand.

ML estimation in the presence of nuisance parameters has typically been addressed in the literature through two different approaches. The first is the so-called stochastic- or unconditional-ML (UML). It assumes the nuisance parameters to be random variables with some a-priori known distribution, and then it takes the marginal of the likelihood function with respect to this prior distribution. The alternative approach is the so-called deterministic- or conditional-ML (CML), which assumes the nuisance parameters to be deterministic unknowns. In CML, the unknown nuisance parameters are estimated from the received samples, and the estimates are substituted back into the likelihood function. To the best of the authors' knowledge, there are no contributions on the application of either the UML or the CML principle to the synchronization of generic FBMC signals. The only similarity can be found in [20], where the CML principle is explicitly applied to the joint blind estimation of time-delay and carrier frequency offset for the very particular case of critically sampled OFDM signals. However, the contribution in [20] disregards the structure of the OFDM signal and it assumes instead that it is formed by a collection of unknown and unstructured Gaussian samples, which are all considered to be nuisance parameters. It is therefore closer to a straightforward extension of the traditional Gaussian approach by Van de Beek in [9], rather than to a truly CML approach, where the only nuisance parameters should be the unknown data symbols and not the whole set of received signal samples.

In this context, the motivation of this section is twofold: First, to introduce both the CML and UML criteria for the synchronization of FBMC signals, and second, to exploit the FBMC signal structure by using the matrix signal model proposed in (19)-(22). To do so, data-only transmissions will be assumed thus leading to nondata-aided (NDA) synchronization schemes. Such approach is of interest when the throughput needs to be maximized, or when very low SNR compromises the performance of decision-directed (DD) approaches [16]. Finally, note that all results presented below are also applicable to the traditional case of single-carrier communication signals by setting $N=1$.

\section{A. CML Approach}

The CML principle is based on assuming the nuisance parameters to be deterministic unknowns. Thus, they can be estimated from the received samples and then the likelihood function can be compressed with respect to the nuisance parameters. This leads to the following estimation criterion:

$$
\widehat{\boldsymbol{\Theta}}_{\mathrm{CML}}=\arg \max _{\boldsymbol{\Theta}} \Lambda(\mathbf{r} ; \boldsymbol{\Theta}, \mathbf{x}=\widehat{\mathbf{x}})
$$

where $\Lambda(\mathbf{r} ; \boldsymbol{\Theta}, \mathbf{x})$ is the likelihood function of the received samples parametrized by the unknowns $\boldsymbol{\Theta}$ and the vector of nuisance parameters, which for the case of NDA synchronization corresponds to the transmitted data symbols $\mathbf{x}$. Because of the Gaussian noise contribution, the likelihood function follows a multivariate Gaussian distribution, $\Lambda(\mathbf{r} ; \boldsymbol{\Theta}, \mathbf{x})=C_{0} \exp \left(-\frac{1}{\sigma_{w}^{2}}\|\mathbf{r}-\mathbf{B}(\boldsymbol{\Theta}) \mathbf{x}\|^{2}\right)$ where $C_{0}$ is an irrelevant constant. Thus, the ML estimate of the unknown data symbols is given by

$$
\widehat{\mathbf{x}}=\left(\mathbf{B}^{H}(\boldsymbol{\Theta}) \mathbf{B}(\boldsymbol{\Theta})\right)^{-1} \mathbf{B}^{H}(\boldsymbol{\Theta}) \mathbf{r} .
$$

It could be argued that the estimate in (24) disregards the discrete nature of the original data symbols, and thus some degradation is incurred. However, the missing gain is often very small, particularly at low- or medium-SNR ranges, and this justifies the common adoption of (24) when compressing the CML cost function in practice. The substitution of (24) into the likelihood function results in the cost function shown below, where the logarithm has been taken and irrelevant constants have been omitted for clarity,

$$
\begin{aligned}
& L_{\mathrm{CML}}^{\prime}(\mathbf{r} \mid \boldsymbol{\Theta}) \\
& \quad=\left\|\mathbf{r}-\mathbf{B}(\boldsymbol{\Theta})\left(\mathbf{B}^{H}(\boldsymbol{\Theta}) \mathbf{B}(\boldsymbol{\Theta})\right)^{-1} \mathbf{B}^{H}(\boldsymbol{\Theta}) \mathbf{r}\right\|^{2} \\
& \quad=\left\|\mathbf{P}_{\mathbf{B}}^{\perp}(\boldsymbol{\Theta}) \mathbf{r}\right\|^{2}
\end{aligned}
$$

with $L_{\mathrm{CML}}^{\prime}(\mathbf{r} \mid \boldsymbol{\Theta}) \doteq-\sigma_{w}^{2} \log \Lambda(\mathbf{r} \mid \boldsymbol{\Theta}, \widehat{\mathbf{x}})$ and $\mathbf{P}_{\mathbf{B}}^{\perp}(\boldsymbol{\Theta}) \doteq \mathbf{I}-$ $\mathbf{B}(\boldsymbol{\Theta})\left(\mathbf{B}^{H}(\boldsymbol{\Theta}) \mathbf{B}(\boldsymbol{\Theta})\right)^{-1} \mathbf{B}^{H}(\boldsymbol{\Theta})$ represents the projection matrix onto the subspace orthogonal to that spanned by the columns 
of $\mathbf{B}(\boldsymbol{\Theta})$. Accordingly, the solution to the CML synchronization of FBMC signals involves a multi-dimensional search

$$
\widehat{\boldsymbol{\Theta}}_{\mathrm{CML}}=\arg \min _{\boldsymbol{\Theta}}\left\|\mathbf{P}_{\mathbf{B}}^{\perp}(\boldsymbol{\Theta}) \mathbf{r}\right\|^{2}
$$

which, unfortunately, requires a significant computational burden. This is in part due to the fact that the cost function in (26) is the most general cost function possible, valid for any kind of FBMC signal in the presence of any channel (either frequency flat or frequency selective). Practical and simpler cost functions should be obtained through the exploitation of the structure of the matrix $\mathbf{B}(\Theta)$, as will be exemplified for the case of critically sampled CP-OFDM in Section IV.

\section{B. Low-SNR UML Approach}

The UML principle is derived by assuming the nuisance parameters to be random variables with some known distribution. In order to remove their presence in the likelihood function, the marginal expectation is taken with respect to the nuisance parameters:

$$
\begin{aligned}
\widehat{\boldsymbol{\Theta}}_{\mathrm{UML}} & =\arg \max _{\boldsymbol{\Theta}} \Lambda(\mathbf{r} ; \boldsymbol{\Theta}) \\
& =\arg \max _{\boldsymbol{\Theta}} \mathrm{E}_{\mathbf{x}}[\Lambda(\mathbf{r} ; \boldsymbol{\Theta}, \mathbf{x})] .
\end{aligned}
$$

Unfortunately, the expectation of the likelihood function often poses unsurmountable obstacles to the derivation of a closedform expression. For this reason, the low-SNR assumption is often adopted in the literature, enabling one to expand the likelihood function into a Taylor series [21, Section 7.5.1] and simplify the expectation with respect to the nuisance parameters. Considering the expansion up to the quadratic term, and retaining only the parameter-dependent terms, we have

$\Lambda(\mathbf{r} \mid \boldsymbol{\Theta}, \mathbf{x}) \stackrel{\sigma_{w}^{2} \rightarrow \infty}{\approx} C_{1}\left[1+\frac{2}{\sigma_{w}^{2}} \chi(\mathbf{r} ; \boldsymbol{\Theta}, \mathbf{x})+\frac{2}{\sigma_{w}^{4}} \chi^{2}(\mathbf{r} ; \boldsymbol{\Theta}, \mathbf{x})\right]$

where $\chi(\mathbf{r} ; \boldsymbol{\Theta} ; \mathbf{x}) \doteq \operatorname{Re}\left[\mathbf{x}^{H} \mathbf{B}^{H}(\boldsymbol{\Theta}) \mathbf{r}\right]-\frac{1}{2}\|\mathbf{B}(\boldsymbol{\Theta}) \mathbf{x}\|^{2}$. If the transmitted symbols are assumed to be zero mean, circularly symmetric with $\mathrm{E}_{\mathbf{x}}\left[\mathbf{x x}^{H}\right]=\mathbf{I}$, then the expectation over $\mathbf{x}$ on both the linear and quadratic terms in (29) results in,

$$
\begin{aligned}
\mathrm{E}_{\mathbf{x}}[\chi(\mathbf{r} ; \boldsymbol{\Theta}, \mathbf{x})]= & -\frac{1}{2} \operatorname{Tr}\{\mathbf{M}(\boldsymbol{\Theta})\} \\
\mathrm{E}_{\mathbf{x}}\left[\chi^{2}(\mathbf{r} ; \boldsymbol{\Theta}, \mathbf{x})\right]= & \frac{1}{2} \mathbf{r}^{H} \mathbf{M}(\boldsymbol{\Theta}) \mathbf{r} \\
& +\frac{1}{4}\|\mathbf{M}(\boldsymbol{\Theta})\|_{F}^{2}+\frac{1}{4} \operatorname{Tr}^{2}\{\mathbf{M}(\boldsymbol{\Theta})\}
\end{aligned}
$$

where $\mathbf{M}(\boldsymbol{\Theta}) \doteq \mathbf{B}(\boldsymbol{\Theta}) \mathbf{B}^{H}(\boldsymbol{\Theta})$. As will be shown later in Section $\mathrm{V}$, the trace of $\mathbf{M}(\boldsymbol{\Theta})$ is a constant term that does not depend on the actual shape of the channel impulse response, but only on its overall energy. Since we are adopting a purely nondata-aided (i.e., blind) approach, there is an inherent scaling ambiguity in the determination of the channel response, and thus, this energy-related constant term in (31) can be ignored. Taking this into account, the low-SNR UML cost function, after applying the logarithm and taking into account that $\log (1+x) \approx x$ for $x \ll 1$, results in

$$
\begin{aligned}
L_{\mathrm{UML}}^{\prime \prime}(\mathbf{r} \mid \boldsymbol{\Theta}) & \approx \sigma_{w}^{4} \log \mathrm{E}_{\mathbf{x}}[\Lambda(\mathbf{r} \mid \boldsymbol{\Theta}, \mathbf{x})] \\
& =\mathbf{r}^{H} \mathbf{M}(\boldsymbol{\Theta}) \mathbf{r}+\frac{1}{2}\|\mathbf{M}(\boldsymbol{\Theta})\|_{F}^{2}
\end{aligned}
$$

and the low-SNR UML estimates become, $\widehat{\Theta}_{\text {low SNR UML }}=$ $\arg \max _{\Theta}\left\{\mathbf{r}^{H} \mathbf{M}(\Theta) \mathbf{r}+\frac{1}{2}\|\mathbf{M}(\Theta)\|_{F}^{2}\right\}$.

\section{BLIND CML SYNCHRONIZATION OF CRITICALLY SAMPLED CP-OFDM SignALS IN SINGLE-PATH CHANNELS}

\section{A. Preliminaries of CP-OFDM Signaling}

The results in Section III provide a general framework for the synchronization of any FBMC signal. In the present section, and as a practical case of interest, these results will be specialized to the blind synchronization of critically sampled CP-OFDM signals. As such, a rectangular pulse shape of $L_{g}=N_{s s}$ samples is adopted and $N=P$. Moreover, the insertion of a cyclic prefix of $N_{s c p}$ samples leads to $N_{s s}=N+N_{s c p}$. Note that the signal model in Section II did not focus on the use of a cyclic prefix; the presence of this prefix was just implicitly modeled through some phase rotations on the transmitted symbols and through an appropriate choice of $N_{s s}>P$. For reasons that will become evident later, it is of use to make the CP explicit in the model and incorporate it within the shaping matrix $\mathbf{A}_{\nu}$. To do so, the $(P \times P)$ IDFT matrix $\mathbf{F}_{P}^{H}$ in (8) needs to be cyclically extended so that its columns have a length of $N_{s s}$ samples. This can be done by making the substitution $\mathbf{F}_{P}^{H} \stackrel{\text { with CP }}{\longrightarrow} \mathbf{T}_{\mathrm{cp}} \mathbf{F}_{P}^{H}$ where the $\left(N_{s s} \times P\right)$ cyclic-prefix matrix $\mathbf{T}_{\mathrm{cp}}$ is defined as,

$$
\mathbf{T}_{\mathrm{cp}} \doteq\left[\begin{array}{cc}
\mathbf{0}_{N_{s c p} \times\left(P-N_{s c p}\right)} & \mathbf{I}_{N_{s c p}} \\
\mathbf{I}_{P} &
\end{array}\right] .
$$

Since $P=N$ in OFDM, the subscript $P$ will be henceforth substituted by $N$ for clarity. The implications on the generic FBMC signal model in (19)-(22) are that, for critically sampled CP-OFDM,

$$
\begin{aligned}
\tilde{\mathbf{F}}^{H} & =\mathbf{1}_{L} \otimes\left(\mathbf{T}_{\mathrm{cp}} \mathbf{F}_{N}^{H}\right) \\
\mathbf{G}_{\nu} & =\operatorname{diag}(\mathbf{e}(\nu, 0)) \mathbf{I}_{L} \otimes \mathbf{1}_{N_{s s}}
\end{aligned}
$$

thus making it possible to simplify the shaping matrix $\mathbf{A}_{\nu}$ to

$$
\mathbf{A}_{\nu}=\operatorname{diag}(\mathbf{e}(\nu, 0))\left[\mathbf{I}_{L} \otimes\left(\mathbf{T}_{\mathrm{cp}} \mathbf{F}_{N}^{H}\right)\right] .
$$

\section{B. CML Orthogonal Projection Matrix for CP-OFDM Signals}

The CML cost function for the problem at hand has been shown in (26) to be given by $L_{\mathrm{CML}}^{\prime}(\mathbf{r} \mid \Theta)=\left\|\mathbf{P}_{\mathrm{B}}^{\perp}(\boldsymbol{\Theta}) \mathbf{r}\right\|^{2}$. Thus, the first step is to calculate the orthogonal projection matrix $\mathbf{P}_{\mathbf{B}}^{\perp}(\boldsymbol{\Theta}) \doteq \mathbf{I}-\mathbf{P}_{\mathbf{B}}(\boldsymbol{\Theta})$, with $\mathbf{P}_{\mathbf{B}}(\boldsymbol{\Theta})=$ $\mathbf{B}(\boldsymbol{\Theta})\left(\mathbf{B}^{H}(\boldsymbol{\Theta}) \mathbf{B}(\boldsymbol{\Theta})\right)^{-1} \mathbf{B}^{H}(\boldsymbol{\Theta})$. Expanding first the inner product in $\mathbf{B}(\boldsymbol{\Theta})$ we have,

$$
\mathbf{B}^{H}(\boldsymbol{\Theta}) \mathbf{B}(\boldsymbol{\Theta})=\mathbf{A}_{\nu}^{H} \mathbf{H}_{\tau}^{H} \mathbf{H}_{\tau} \mathbf{A}_{\nu}=E_{h} \mathbf{A}_{\nu}^{H} \mathbf{A}_{\nu}
$$


which is found to depend on the structure of the transmitted signal according to the shaping matrix $\mathbf{A}_{\nu}$ only. This is true since for single-path channels, $\mathbf{H}_{\tau}^{H} \mathbf{H}_{\tau}=E_{h} \mathbf{I}_{Q}$ with $E_{h} \doteq$ $\sum_{m=-M_{h}}^{M_{h}}|h(m)|^{2}$, thanks to the autocorrelation properties of the sinc function and provided that $M_{h}$ is large enough. With $\mathbf{A}_{\nu}$ in its more general form in (21), the inner product $\mathbf{A}_{\nu}^{H} \mathbf{A}_{\nu}$ results in an $(L N \times L N)$ block matrix whose individual $(N \times N)$ submatrices are shown in Appendix A to be given by

$$
\mathbf{D}_{l_{1}, l_{2}}=\tilde{\mathbf{F}} \operatorname{diag}\left(\mathbf{g}_{l_{1}}(\boldsymbol{\Theta}) \odot \mathbf{g}_{l_{2}}^{*}(\boldsymbol{\Theta})\right) \tilde{\mathbf{F}}^{H}
$$

with $\left\{l_{1}, l_{2}\right\}=-K, \ldots, K$. For the particular case of CP-OFDM signals, the adoption of rectangular and non-overlapping pulses leads the Hadamard product $\mathbf{g}_{l_{1}}(\boldsymbol{\Theta}) \odot \mathbf{g}_{l_{2}}^{*}(\boldsymbol{\Theta})$ to be zero except for $l_{1}=l_{2}$. As a result, for CP-OFDM the inner product $\mathbf{A}_{\nu}^{H} \mathbf{A}_{\nu}$ becomes a block diagonal matrix as follows 2 :

$$
\begin{aligned}
\mathbf{A}_{\nu}^{H} \mathbf{A}_{\nu} & =\mathbf{I}_{L} \otimes\left[\mathbf{F}_{N} \mathbf{T}_{\mathrm{cp}}^{H} \mathbf{T}_{\mathrm{cp}} \mathbf{F}_{N}^{H}\right] \\
& =\mathbf{I}_{L} \otimes\left[\mathbf{F}_{N}\left(\mathbf{I}_{N}+\boldsymbol{\Lambda}_{N_{s c p}}\right) \mathbf{F}_{N}^{H}\right]
\end{aligned}
$$

where $\boldsymbol{\Lambda}_{N s c p} \doteq \operatorname{diag}\left(\left[\mathbf{0}_{N-N_{s c p}}^{T} \mathbf{1}_{N s c p}^{T}\right]^{T}\right)$ is a diagonal matrix whose last $N_{s c p}$ samples are equal to 1 . These last samples can therefore be understood as a rectangular pulse shape of length $N_{s c p}$. Note also that the result in (40) no longer depends on the carrier frequency error $\nu$.

The inverse required in the projection matrix $\mathbf{P}_{\mathbf{B}}(\boldsymbol{\Theta})$ can easily be calculated once $\mathbf{A}_{\nu}^{H} \mathbf{A}_{\nu}$ and thus $\mathbf{B}^{H}(\boldsymbol{\Theta}) \mathbf{B}(\boldsymbol{\Theta})$ are found to be block diagonal. In that case,

$$
\left(\mathbf{B}^{H}(\boldsymbol{\Theta}) \mathbf{B}(\boldsymbol{\Theta})\right)^{-1}=\frac{1}{E_{h}} \mathbf{I}_{L} \otimes\left[\mathbf{F}_{N}\left(\mathbf{I}_{N}+\boldsymbol{\Lambda}_{N_{s c p}}\right) \mathbf{F}_{N}^{H}\right]^{-1} .
$$

Applying the Woodbury inversion lemma and taking into account that $\mathbf{F}_{N} \mathbf{F}_{N}^{H}=\mathbf{I}_{N}$, we get

$$
\left(\mathbf{B}^{H}(\boldsymbol{\Theta}) \mathbf{B}(\boldsymbol{\Theta})\right)^{-1}=\frac{1}{E_{h}} \mathbf{I}_{L} \otimes\left[\mathbf{I}_{N}-\frac{1}{2} \mathbf{F}_{N} \boldsymbol{\Lambda}_{N_{s c p}} \mathbf{F}_{N}^{H}\right] .
$$

This result can then be substituted back into projection matrix $\mathbf{P}_{\mathbf{B}}$, which after some straightforward manipulations is found to result in,

$$
\begin{aligned}
\mathbf{P}_{\mathbf{B}}(\boldsymbol{\Theta}) \\
=\frac{1}{E_{h}} \mathbf{B}(\boldsymbol{\Theta})\left[\mathbf{I}_{L} \otimes\left[\mathbf{I}_{N}-\frac{1}{2} \mathbf{F}_{N} \boldsymbol{\Lambda}_{N_{s c p}} \mathbf{F}_{N}^{H}\right]\right] \mathbf{B}^{H}(\boldsymbol{\Theta}) \\
=\frac{1}{E_{h}} \mathbf{H}_{\tau} \operatorname{diag}(\mathbf{e}(\nu, 0))\left[\mathbf{I}_{L} \otimes\left(\mathbf{T}_{\mathrm{cp}} \mathbf{F}_{N}^{H}\right)\right] \\
\quad \times\left[\mathbf{I}_{L} \otimes\left[\mathbf{I}_{N}-\frac{1}{2} \mathbf{F}_{N} \boldsymbol{\Lambda}_{N_{s c p}} \mathbf{F}_{N}^{H}\right]\right] \\
\\
\cdot\left[\mathbf{I}_{L} \otimes\left(\mathbf{F}_{N} \mathbf{T}_{\mathrm{cp}}^{H}\right)\right] \operatorname{diag}\left(\mathbf{e}^{*}(\nu, 0)\right) \mathbf{H}_{\tau}^{H} \\
=\frac{1}{E_{h}} \mathbf{H}_{\tau}\left[\mathbf{I}_{L} \otimes\left(\left[\mathbf{T}_{\mathrm{cp}}\left(\mathbf{I}_{N}-\frac{1}{2} \boldsymbol{\Lambda}_{N_{s c p}}\right) \mathbf{T}_{\mathrm{cp}}^{H}\right] \odot \mathbf{V}_{N, \nu}\right)\right] \mathbf{H}_{\tau}^{H} .
\end{aligned}
$$

The result in (46) depends on the carrier frequency error through the Toeplitz matrix $\mathbf{V}_{N, \nu}$ defined as,

$$
\left[\mathbf{V}_{N, \nu}\right]_{p, q} \doteq e^{j \frac{2 \pi}{N}(p-q) \nu} .
$$

\footnotetext{
${ }^{2}$ The same result is obtained if the inner-product $\mathbf{A}_{\nu}^{H} \mathbf{A}_{\nu}$ is directly calculated using the simplified shaping matrix in (36).
}

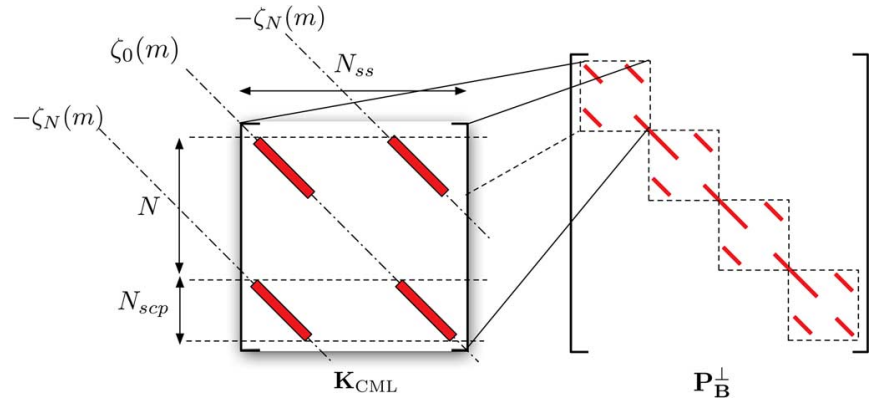

Fig. 1. Illustration of the kernel matrix $\mathbf{K}_{\mathrm{CML}}$ in (50) and the periodic structure of $\mathbf{P}_{\mathbf{B}}^{\perp}$ in (49) for critically sampled CP-OFDM signals. For the sake of clarity, $\mathbf{P}_{\mathrm{B}}^{\perp}$ has been depicted for a single-path channel with $\{\tau=0, \nu=0\}$. In the presence of time-delay, the entries of $\mathbf{P}_{\mathrm{B}}^{\perp}$ move in a diagonal-wise manner by an amount equal to $\tau$. In the presence of a frequency error, each diagonal of $\mathbf{P}_{\mathbf{B}}^{\perp}$ is multiplied by a constant complex number, thus still preserving the same periodic structure.

The orthogonal projection matrix required in the CML cost function can thus be obtained as $\mathbf{P}_{\mathbf{B}}^{\perp}(\boldsymbol{\Theta})=\mathbf{I}_{Q}-\mathbf{P}_{\mathbf{B}}(\boldsymbol{\Theta})$. If the observation interval is asymptotically large (i.e., $L \rightarrow \infty$ ) the outer-product of the single-path channel matrix can be fairly approximated as $\mathbf{H}_{\tau} \mathbf{H}_{\tau}^{H} \approx E_{h} \mathbf{I}_{Q}$ and thus the orthogonal projection matrix projection results in

$$
\begin{aligned}
& \mathbf{P}_{\mathbf{B}}^{\perp}(\boldsymbol{\Theta}) \\
& \approx \frac{1}{E_{h}} \mathbf{H}_{\tau} \mathbf{H}_{\tau}^{H}-\frac{1}{E_{h}} \mathbf{H}_{\tau} \\
& \quad \times\left[\mathbf{I}_{L} \otimes\left(\left[\mathbf{T}_{\mathrm{cp}}\left(\mathbf{I}_{N}-\frac{1}{2} \boldsymbol{\Lambda}_{N_{s c p}}\right) \mathbf{T}_{\mathrm{cp}}^{H}\right] \odot \mathbf{V}_{N, \nu}\right)\right] \mathbf{H}_{\tau}^{H} \\
& =\frac{1}{E_{h}} \mathbf{H}_{\tau}\left(\mathbf{I}_{L} \otimes \mathbf{K}_{\mathrm{CML}}\right) \mathbf{H}_{\tau}^{H}
\end{aligned}
$$

where $\mathbf{K}_{\mathrm{CML}}$ is a $\left(N_{s s} \times N_{s s}\right)$ Toeplitz matrix that is henceforth referred to as the kernel matrix for the CML synchronization criterion, and is defined as follows,

$$
\mathbf{K}_{\mathrm{CML}} \doteq\left[\mathbf{I}_{N_{s s}}-\mathbf{T}_{\mathrm{cp}}\left(\mathbf{I}_{N}-\frac{1}{2} \boldsymbol{\Lambda}_{N_{s c p}}\right) \mathbf{T}_{\mathrm{cp}}^{H}\right] \odot \mathbf{V}_{N, \nu}
$$

The kernel matrix in (50) summarizes the cyclostationary structure contained within the transmitted signal. This periodic behavior is indeed what allows the receiver to estimate the synchronization parameters in a nondata-aided manner. The particular structure of the kernel matrix $\mathbf{K}_{\mathrm{CML}}$ and the induced cyclostationarity on the orthogonal projection matrix $\mathbf{P}_{\mathbf{B}}^{\perp}(\boldsymbol{\Theta})$ are illustrated in Fig. 1. In view of this structure, it is convenient to express $\mathbf{P}_{\mathbf{B}}^{\perp}(\boldsymbol{\Theta})$ in a diagonal-wise manner. To do so, let $\left[\mathbf{P}_{\mathbf{B}}^{\perp}(\boldsymbol{\Theta})\right]_{m+u, m}$ be the $m$-th entry along the $u$-th diagonal of $\mathbf{P}_{\mathbf{B}}^{\perp}(\boldsymbol{\Theta})$. Then, for a single-path channel,

$$
\begin{aligned}
& {\left[\mathbf{P}_{\mathbf{B}}^{\perp}(\boldsymbol{\Theta})\right]_{m+u, m}=-\delta(u-N) \tilde{\zeta}_{N}(m-\tau) e^{j \frac{2 \pi}{N} \nu u}} \\
& \quad-\delta(u+N) \tilde{\zeta}_{N}(m-\tau) e^{-j \frac{2 \pi}{N} \nu u}+\delta(u) \tilde{\zeta}_{0}(m-\tau)
\end{aligned}
$$

where $\tilde{\zeta}_{0}(m) \doteq \sum_{l=-K}^{K} \zeta_{0}\left(m-l N_{s s}\right)$ and $\tilde{\zeta}_{N}(m) \doteq$ $\sum_{l=-K}^{K} \zeta_{N}\left(m-l N_{s s}\right)$ are $N_{s s}$-periodic signals (in the limit of $K \rightarrow \infty$ ) whose basic waveforms are given by,

$$
\begin{aligned}
\zeta_{0}(m) & \doteq \frac{1}{2}\left[\operatorname{rect}_{N_{s c p}}(m)+\operatorname{rect}_{N_{s c p}}(m-N)\right] \\
\zeta_{N}(m) & \doteq \frac{1}{2} \operatorname{rect}_{N_{s c p}}(m)
\end{aligned}
$$


with $m=0, \ldots, N_{s s}-1$ and rect $N_{s c p}(x)$ defined as a rectangular pulse of $N_{s c p}$ samples when $x$ is an integer, or its corresponding interpolated version when $x$ is a non-integer.

\section{Blind CML Joint Time-Delay and Frequency Estimator}

The result for the orthogonal projection matrix $\mathbf{P}_{\mathbf{B}}^{\perp}(\boldsymbol{\Theta})$ in (49) is of paramount importance for understanding how the signal design for CP-OFDM has a direct impact on the way that blind synchronization can be implemented. The resulting structure in $\mathbf{P}_{\mathrm{B}}^{\perp}(\boldsymbol{\Theta})$ can then be exploited to allow a simple derivation of the CML cost function, which can be expanded into scalar form as follows,

$$
\begin{aligned}
L_{\mathrm{CML}}^{\prime}(\mathbf{r} \mid \boldsymbol{\Theta}) & \left\|\mathbf{P}_{\mathbf{B}}^{\perp}(\boldsymbol{\Theta}) \mathbf{r}\right\|^{2}=\operatorname{Tr}\left\{\mathbf{P}_{\mathbf{B}}^{\perp}(\boldsymbol{\Theta}) \mathbf{r r}^{H}\right\} \\
= & \sum_{m=-M}^{M}\left[\mathbf{P}_{\mathbf{B}}^{\perp}(\boldsymbol{\Theta})\right]_{m, m}\left[\mathbf{r} \mathbf{r}^{H}\right]_{m, m} \\
& +\sum_{u=1}^{Q_{r}-1} \sum_{m=-M}^{M-u}\left[\mathbf{P}_{\mathbf{B}}^{\perp}(\boldsymbol{\Theta})\right]_{m+u, m}\left[\mathbf{r r}^{H}\right]_{m+u, m} \\
& +\sum_{u=-Q_{r}+1}^{-1} \sum_{m=-M+|u|}^{M}\left[\mathbf{P}_{\mathbf{B}}^{\perp}(\boldsymbol{\Theta})\right]_{m+u, m}\left[\mathbf{r r}^{H}\right]_{m+u, m} \\
& \stackrel{N}{=} \sum_{m=-M}^{M} \tilde{\zeta}_{0}(m-\tau)|r(m)|^{2} \\
& -2 \operatorname{Re}\left[e^{j 2 \pi \nu} \sum_{m=-M}^{M} \tilde{\zeta}_{N}(m-\tau) r(m+N) r^{*}(m)\right] .
\end{aligned}
$$

As can be seen, the blind CML cost function depends on the time-delay and the carrier frequency error, and the latter can be estimated once the time-delay estimate is available as follows,

$$
\widehat{\nu}=\frac{1}{2 \pi} \arg \left\{\sum_{m=-M}^{M} \tilde{\zeta}_{N}(m-\tau) r(m+N) r^{*}(m)\right\} .
$$

It still remains how to implement this estimator in an efficient manner and what the implications are of the operations required to perform (57). It is important to note that the range of values for this estimator is $\hat{\nu} \in[-1 / 2,+1 / 2)$, which is $P$ times smaller than the initial full range defined for $\nu$ in (2). Since $\nu \doteq f_{\epsilon} / F_{0}$, this implies that $\widehat{f}_{\epsilon} \in\left[-F_{0} / 2,+F_{0} / 2\right)$. This constraint is common to most frequency estimators for MC signals, and it requires the frequency error to lie within a fraction of the carrier spacing for the carrier estimates to be unbiased.

Let us proceed with the derivation by compressing the estimate for $\nu$ in (57) into the blind CML cost function in (56), thus leading to a function depending on the time-delay only. Following this approach it is not difficult to see that

$$
\begin{aligned}
L_{\mathrm{CML}}^{\prime}(\mathbf{r} \mid \tau)= & \sum_{m=-M}^{M} \tilde{\zeta}_{0}(m-\tau)|r(m)|^{2} \\
& -2\left|\sum_{m=-M}^{M} \tilde{\zeta}_{N}(m-\tau) r(m+N) r^{*}(m)\right| .
\end{aligned}
$$

To exploit the periodic behavior of $\tilde{\zeta}_{N}(m)$ and $\tilde{\zeta}_{0}(m)$, we decompose the time index as $m=p N_{s s}+q$ with $p=-K, \ldots, K$ and $q=0, \ldots, N_{s s}-1$. Then the blind CML cost function can be rearranged as

$$
\begin{aligned}
L_{\mathrm{CML}}^{\prime}(\mathbf{r} \mid \tau)= & \sum_{p=-K}^{K} \sum_{q=0}^{N_{s s}-1} \sum_{l=-K}^{K} \zeta_{0}\left((p-l) N_{s s}\right. \\
& +q-\tau)\left|r^{*}\left(p N_{s s}+q\right)\right|^{2} \\
& -2 \mid \sum_{p=-K}^{K} \sum_{q=0}^{N_{s s}-1} \sum_{l=-K}^{K} \zeta_{N}\left((p-l) N_{s s}\right. \\
& +q-\tau) r\left(p N_{s s}+q+N\right) r^{*}\left(p N_{s s}+q\right) \mid .
\end{aligned}
$$

Because of the finite time support of $\zeta_{N}(m)$ and $\zeta_{0}(m)$, only those terms with $l=p$ need to be kept. Furthermore, special care must be taken with the periodic structure of $\tilde{\zeta}_{N}(m)$ and $\tilde{\zeta}_{0}(m)$, which translates into a circular structure in their basic waveforms $\zeta_{N}(m)$ and $\zeta_{0}(m)$ when a time-delay $\tau$ is present. Such a $N_{s s}$-circular structure will be denoted by $\zeta_{0}(m-\tau)_{N_{s s}}$ and $\zeta_{N}(m-\tau)_{N_{s s}}$, respectively. With these considerations, the expression above simplifies to

$$
\begin{aligned}
L_{\mathrm{CML}}^{\prime}(\mathbf{r} \mid \tau)= & \sum_{p=-K}^{K} \sum_{q=0}^{N_{s s}-1} \zeta_{0}(q-\tau)_{N_{s s}}\left|r^{*}\left(p N_{s s}+q\right)\right|^{2} \\
& -2 \mid \sum_{p=-K}^{K} \sum_{q=0}^{N_{s s}-1} \zeta_{N}(q-\tau)_{N_{s s}} \\
& \times r\left(p N_{s s}+q+N\right) r^{*}\left(p N_{s s}+q\right) \mid \\
= & \sum_{q=0}^{N_{s s}-1} \zeta_{0}(q-\tau)_{N_{s s}} \tilde{R}_{r}(q ; 0) \\
& -2\left|\sum_{q=0}^{N_{s s}-1} \zeta_{N}(q-\tau)_{N_{s s}} \tilde{R}_{r}(q ; N)\right|
\end{aligned}
$$

where

$$
\tilde{R}_{r}(k ; u) \doteq \sum_{n=-\infty}^{\infty} R_{r}\left(k-n N_{s s} ; u\right)
$$

with $R_{r}(k ; u) \doteq r(k+u) r^{*}(k)$ is the time-varying synchronous autocorrelation of the received samples and $k=0, \ldots, N_{s s}-$ 1. Finally, the CML time-delay estimation can be expressed in compact form as,

$$
\hat{\tau}_{\mathrm{CML}}=\arg \max _{\tau}\left\{\left|y_{N}(\tau)\right|-\frac{1}{2} y_{0}(\tau)\right\}
$$

where

$$
\begin{aligned}
y_{N}(\tau) & \doteq\left[\zeta_{N}(-m-\tau) \circledast_{N_{s s}} \tilde{R}_{r}(m ; N)\right]_{m=0} \\
y_{0}(\tau) & \doteq\left[\zeta_{0}(-m-\tau) \circledast_{N_{s s}} \tilde{R}_{r}(m ; 0)\right]_{m=0}
\end{aligned}
$$

and $\circledast_{N_{s s}}$ represents the circular convolution of length $N_{s s}$. Consequently, the CML carrier frequency estimation in (57) simplifies to the following expression,

$$
\hat{\nu}_{\mathrm{CML}}=\frac{1}{2 \pi} \arg \left\{y_{N}\left(\hat{\tau}_{\mathrm{CML}}\right)\right\} \text {. }
$$




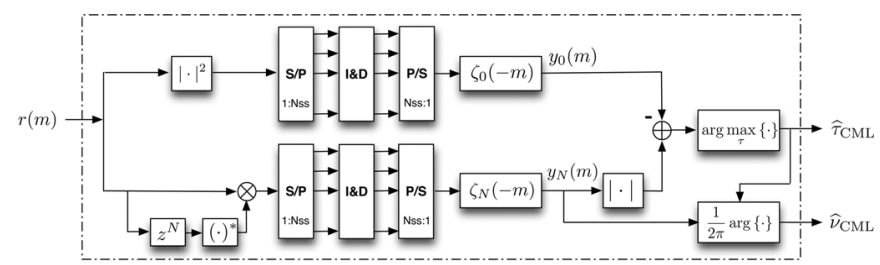

Fig. 2. Architecture of the CML joint time-delay and frequency estimator for critically sampled CP-OFDM signals.

A proposed implementation of the CML estimator derived herein for the case of critically sampled CP-OFDM signals is depicted in Fig. 2. The blocks indicated by "S/P" and " $\mathrm{P} / \mathrm{S}$ " are serial-to-parallel and parallel-to-serial converters that take advantage of the $N_{s s}$-samples cyclic structure of the CP-OFDM signal. The block indicated by "I\&D" is an integrate-and-dump operation that accumulates $L$ vectors of samples and then provides the result at its output. Finally, the blocks with $\zeta_{0}(-m)$ and $\zeta_{N}(-m)$ perform the circular convolution, as denoted by (64)-(65).

It is interesting to note that the resulting CML cost function in (61) embraces the one proposed in [22] for the synchronization of a voice-band modem that exploits the presence of a cyclic sequence. Although originally proposed in an ad-hoc manner, this same cost function was later obtained under the Minimum Mean Square Error criterion in [23], where the estimation of time-delay and frequency error was addressed for an OFDM signal modeled as a Gaussian random process with known covariance matrix. ML estimation under the Gaussian assumption was considered in the work by Van de Beek in [9], and the resulting estimator is also found to coincide with the one in (63)-(66), except for the fact that Van de Beek's estimator has an SNR dependent factor $\rho \doteq \mathrm{SNR} /(\mathrm{SNR}+1)$ multiplying the second term in (63). As a consequence, Van de Beek's estimator requires the SNR to be known a-priori or to be calculated online, which further complicates the implementation compared to the proposed estimator in (63)-(66). It has been reported in [23] that the advantage of including this SNR-dependent factor yields a slight performance improvement, particularly in low-SNR conditions and for some multipath channels, when compared to the MMSE criterion (and thus to the CML criterion, since both have been shown to coincide). For high-SNR, $\rho \rightarrow 1$ and thus all estimators converge to the same expression without incurring any penalty. This observation is consistent with the CML framework considered herein, since $\widehat{\mathbf{x}} \rightarrow \mathbf{x}$ in the high-SNR region and thus CML operates with perfect side information and provides optimal performance. For the low-SNR regime, no optimality can be expected from CML since $\widehat{\mathbf{x}}$ diverges from $\mathbf{x}$. Therefore, the criterion is forced to operate with imperfect side information and some performance loss is incurred. This effect will motivate the formulation of a novel low-SNR optimal estimator in the next section, and its application to both single- and multi-path channels.

\section{BLIND LOW-SNR UML SYNCHRONIZATION OF CRITICALLY SAMPLED CP-OFDM SIGNALS}

One of the main problems of the CML estimation criterion is the matrix inversion required for implementing the orthogonal projection matrix in (27). In the previous section dealing with single-path channels, this problem was circumvented because of the diagonal- and block-wise structure of the signal matrix

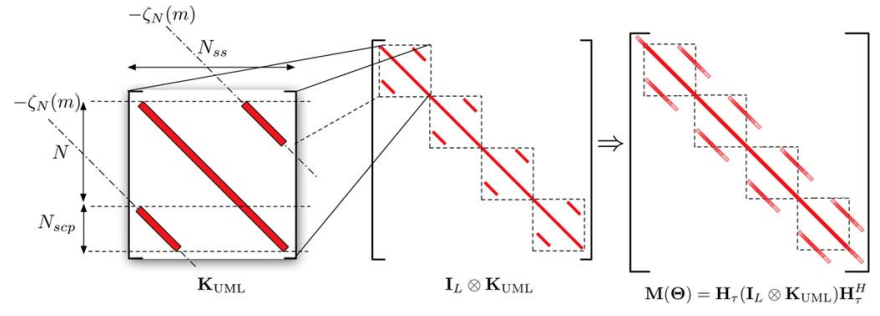

Fig. 3. Illustration of the periodic structure exhibited by the kernel matrix $\mathbf{K}_{\mathrm{UML}}$ and the resulting block-matrix $\mathbf{M}(\boldsymbol{\Theta})$ appearing in the low-SNR UML cost function in (67). Note that even in the presence of multipath (see picture on the right hand side), the entries of $\mathbf{M}(\Theta)$ are spread in a diagonal-wise manner, thus still preserving the same diagonal structure.

in (41) to be inverted. That property allowed us to easily calculate the inverse in an analytical manner and thus proceed with the derivation of the CML synchronizer. Unfortunately, such a nice diagonal-wise structure is lost in the presence of multipath channels. In this case, it is preferrable to explore new ways to solve the ML synchronization problem, and it is here where the low-SNR UML criterion plays an important role. First of all, let us recall its cost function from (32), which reads

$$
L_{\mathrm{UML}}^{\prime \prime}(\mathrm{r} \mid \boldsymbol{\Theta})=\mathbf{r}^{H} \mathbf{M}(\boldsymbol{\Theta}) \mathbf{r}+\frac{1}{2}\|\mathbf{M}(\boldsymbol{\Theta})\|_{F}^{2} .
$$

The next step is to analyze the structure of $\mathbf{M}(\boldsymbol{\Theta})$ in order to understand its implications on the cost function in (67). Being defined in Section III-B as $\mathbf{M}(\boldsymbol{\Theta}) \doteq \mathbf{B}(\boldsymbol{\Theta}) \mathbf{B}^{H}(\boldsymbol{\Theta})$, it can be further expanded in a similar manner as was previously done when calculating the projection matrix in (43). Substituting $\mathbf{B}(\boldsymbol{\Theta})$ in (20) and using (36), we have

$$
\begin{aligned}
\mathbf{M}(\boldsymbol{\Theta})= & \mathbf{H}_{\tau} \mathbf{A}_{\nu} \mathbf{A}_{\nu}^{H} \mathbf{H}_{\tau}^{H} \\
= & \mathbf{H}_{\tau} \operatorname{diag}(\mathbf{e}(\nu, 0))\left[\mathbf{I}_{L} \otimes\left(\mathbf{T}_{\mathrm{cp}} \mathbf{F}_{N}^{H}\right)\right] \\
& \times\left[\mathbf{I}_{L} \otimes\left(\mathbf{F}_{N} \mathbf{T}_{\mathrm{cp}}^{H}\right)\right] \operatorname{diag}\left(\mathbf{e}^{*}(\nu, 0)\right) \mathbf{H}_{\tau}^{H} \\
= & \mathbf{H}_{\tau}\left(\mathbf{I}_{L} \otimes \mathbf{K}_{\mathrm{UML}}\right) \mathbf{H}_{\tau}^{H}
\end{aligned}
$$

where $\mathbf{K}_{\mathrm{UML}}$ is a $\left(N_{s s} \times N_{s s}\right)$ Toeplitz matrix similar to that for the CML case, and is henceforth referred to as the kernel matrix for the low-SNR UML synchronization criterion. This matrix is defined as

$$
\mathbf{K}_{\mathrm{UML}} \doteq\left[\mathbf{T}_{\mathrm{cp}} \mathbf{T}_{\mathrm{cp}}^{H}\right] \odot \mathbf{V}_{N, \nu}
$$

and it is affected in a diagonal-wise manner by the Toeplitz matrix $\mathbf{V}_{N, \nu}$ defined in (47). The kernel matrix in (71) summarizes the cyclostationary structure within the CP-OFDM signal that is relevant for synchronization purposes in the low-SNR regime. The structure of this matrix is indeed very similar to the one in the CML case, as it can be observed in the left hand side of Fig. 3.

The only difference with respect to the CML case is that the main diagonal of $\mathbf{K}_{\mathrm{UML}}$ is now constant, and thus, for an asymptotically large observation interval, it does not convey any information regarding the synchronization parameters. This has two main implications. The first is that we should discard the correlation lag $u=0$ of the input samples when operating in the low-SNR regime, the correlation lag which is indeed the most degraded by the presence of noise. The second implication, closely related to the first one, is that $\operatorname{Tr}\{\mathbf{M}(\Theta)\}$ turns out to be a constant scaling factor that only depends on the overall 
channel energy and not on its particular shape. Therefore, it can be ignored in the low-SNR UML terms in (31), thus confirming the validity of the low-SNR UML cost function in (32) and (67).

Alternatively, and following the same approach as in Section IV-C, the low-SNR UML cost function can be decomposed into scalar form as follows,

$$
\begin{array}{r}
L_{\mathrm{UML}}^{\prime \prime}(\mathbf{r} \mid \boldsymbol{\Theta})=2 \operatorname{Re}\left[e^{j 2 \pi \nu} \sum_{q=0}^{N_{s s}-1} \zeta_{N}(q ; \boldsymbol{\Theta})_{N_{s s}} \tilde{R}_{r}(q ; N)\right] \\
+L \sum_{q=0}^{N_{s s}-1}\left|\zeta_{N}(q ; \boldsymbol{\Theta})_{N_{s s}}\right|^{2}+C_{0}
\end{array}
$$

where $\zeta_{N}(q ; \Theta)_{N_{s s}}$ is the basic (and unknown) waveform of length $N_{s s}$ being repeated along the $N$-th diagonal of $\mathbf{M}(\boldsymbol{\Theta})$, and dependent on the channel parameters through $\boldsymbol{\Theta}$. In (72), it has been taken into account that just the $\pm N$-th diagonals of $\mathbf{M}(\boldsymbol{\Theta})$ are relevant for the problem at hand, and that $\|\mathbf{M}(\boldsymbol{\Theta})\|_{F}^{2}=\sum_{u=-Q+1}^{Q-1} \sum_{m=-M}^{M-u}\left|[\mathbf{M}(\boldsymbol{\Theta})]_{m+u, m}\right|^{2}=$ $2 L \sum_{m=0}^{N_{s s}-1}\left|\zeta_{N}(m ; \boldsymbol{\Theta})\right|^{2}+C_{0}$, with $C_{0}$ an irrelevant constant given by the energy along the principal diagonal of $\mathbf{M}(\Theta)$.

\section{A. Low-SNR UML Estimator for Single-Path Channels}

The derivation of the low-SNR UML joint time-delay estimator is straightforward based on the similarities between the cost function in (67) and the CML counterpart $L_{\mathrm{CML}}^{\prime}(\mathbf{r} \mid \Theta)=$ $\left\|\mathbf{P}_{\mathbf{B}}^{\perp}(\boldsymbol{\Theta}) \mathbf{r}\right\|^{2}=\mathbf{r}^{H} \mathbf{P}_{\mathbf{B}}^{\perp}(\boldsymbol{\Theta}) \mathbf{r}$ in (26). The main difference is the presence of $\|\mathbf{M}(\boldsymbol{\Theta})\|_{F}^{2}$ in (67), but this term can be ignored for single-path channels because it is constant. Indeed, a change in $\tau$ just implies moving the entries of $\mathbf{M}(\boldsymbol{\Theta})$ in a diagonal-wise manner so that, asymptotically, the Frobenius norm remains the same $^{3}$. Ignoring the last term in (67), we essentially have the same type of single-term quadratic cost function as in the CML case. At this point the only difference between both criteria lies in the different types of kernel matrices they use. In spite of this, the low-SNR UML kernel matrix is essentially the same as the CML one, except for the fact that the main diagonal is now constant, and therefore it can be ignored for synchronization purposes. As a result, the very same architecture can be considered here, with the only modification that the first branch implementing the correlation lag $u=0$ can be removed. This leads to the following low-SNR UML joint time-delay and frequency estimator:

$$
\begin{aligned}
& \hat{\tau}_{\mathrm{UML}}=\arg \max _{\tau}\left\{\left|y_{N}(\tau)\right|\right\} \\
& \hat{\nu}_{\mathrm{UML}}=\frac{1}{2 \pi} \arg \left\{y_{N}\left(\hat{\tau}_{\mathrm{UML}}\right)\right\}
\end{aligned}
$$

where $y_{N}(\tau)$ is defined in (64) and the resulting architecture is depicted in Fig. 4.

Similar to what already discussed at the end of Section IV-C, the low-SNR UML estimator obtained in (73) is found to coincide with Beek's ML estimator, but for the low-SNR regime where $\rho \rightarrow 0$. This observation confirms the optimality of the proposed low-SNR UML estimator in the low-SNR regime.

\footnotetext{
${ }^{3} \mathrm{~A}$ change in $\nu$ does not change the Frobenius norm either, because the frequency error appears as just a complex constant along each diagonal of $\mathbf{M}(\Theta)$. Nevertheless, we keep the term $\|\mathbf{M}(\boldsymbol{\Theta})\|_{F}^{2}$ in the general expression for the low-SNR UML cost function in (67) because it will play a relevant role later on, when we cope with multipath channels.
}

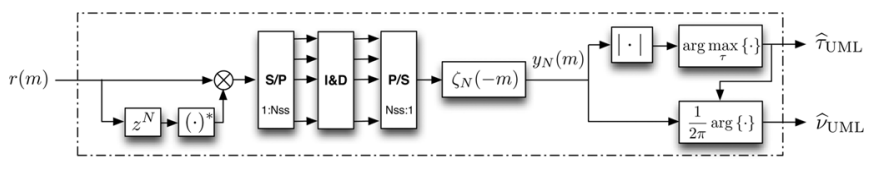

Fig. 4. Architecture of the UML estimator in (73)-(74) for critically sampled CP-OFDM signals and single-path channels.

For the high-SNR regime, no optimality can be guaranteed because the power-related term $y_{0}(\tau)$ is not present in (73). However, $y_{0}(\tau)$ is approximately constant over $\tau$ provided that the number of OFDM symbols $L$ is large enough. In that case, the CML estimator in (63) coincides with the low-SNR UML estimator in (73), and it could be claimed that the low-SNR UML estimator is asymptotically optimal (i.e., $L \rightarrow \infty$ ) for the high-SNR regime, too. This is the underlying reason for the good performance exhibited by time-delay estimators based on (73), which have been widely adopted by many authors although initially proposed in an ad-hoc manner (e.g., [24], [25]).

\section{B. Extension of the Low-SNR UML Estimator to Multipath Channels}

Contrary to the CML case, a step further can easily be taken for the low-SNR UML criterion in order to explicitly incorporate the presence of multipath channel into the formulation. This is certainly a key novelty of the present work, taking one step forward with respect to the current state-of-the-art on blind OFDM synchronization, where the common approach is to ignore the actual channel impulse response within the algorithm derivation (see for instance [15], [26]-[28] or the landmark work in [8], [9]). This is in contrast to pilot-assisted techniques, which have been widely studied in the literature, and for which channel estimation is indeed a constituent part of any time-delay estimation algorithm.

In order to incorporate the multipath channel into the problem under study, the cost function is basically the same as the one already introduced in (67) or (72), but some remarks must be made regarding the structure of $\mathbf{M}(\Theta)$ due to the presence of multipath. Using the matrix decomposition in (18), we have

$$
\begin{aligned}
\mathbf{M}(\boldsymbol{\Theta})= & \sum_{i=0}^{L_{h}-1} \sum_{j=0}^{L_{h}-1} h_{i} h_{j}^{*} \mathbf{H}_{i, \tau}\left(\mathbf{I}_{L} \otimes \mathbf{K}_{\mathrm{UML}}\right) \mathbf{H}_{j, \tau}^{H} \\
= & \sum_{i=0}^{L_{h}-1}\left|h_{i}\right|^{2} \mathbf{H}_{i, \tau}\left(\mathbf{I}_{L} \otimes \mathbf{K}_{\mathrm{UML}}\right) \mathbf{H}_{i, \tau}^{H} \\
& +\sum_{i \neq j} h_{i} h_{j}^{*} \mathbf{H}_{i, \tau}\left(\mathbf{I}_{L} \otimes \mathbf{K}_{\mathrm{UML}}\right) \mathbf{H}_{j, \tau}^{H} \\
& \stackrel{L_{h} \gg 1}{\approx} \sum_{i=0}^{L_{h}-1}\left|h_{i}\right|^{2} \mathbf{H}_{i, \tau}\left(\mathbf{I}_{L} \otimes \mathbf{K}_{\mathrm{UML}}\right) \mathbf{H}_{i, \tau}^{H} .
\end{aligned}
$$

The approximation in (77) is reasonable for dense multipath conditions with uncorrelated scattering, as the second term in (76) can be neglected in virtue of the law of large numbers. In these circumstances, $\mathbf{M}(\Theta)$ can be expressed as a linear combination of the single-path contributions $\mathbf{H}_{i, \tau}\left(\mathbf{I}_{L} \otimes \mathbf{K}_{\mathrm{UML}}\right) \mathbf{H}_{i, \tau}^{H}$, which interestingly all preserve the same diagonal-wise structure already highlighted for the single-path channel. An example is depicted in the right hand side of Fig. 3, where $\mathbf{H}_{\tau}\left(\mathbf{I}_{L} \otimes\right.$ $\left.\mathbf{K}_{\mathrm{UML}}\right) \mathbf{H}_{\tau}$ is depicted in the presence of a multipath channel. 
As it can be seen in the figure, the effect of multipath is to spread the entries along both the upper and lower $N$-th diagonal of $\mathbf{M}(\Theta)$. Note that no overlap is incurred along these diagonals because $N_{s s}$ is always greater than or equal to $2 N_{s c p}$. These spread diagonal entries continue to exhibit $N_{s s}$-sample periodicity, but the waveform being repeated turns out to be the convolution of the original rectangular pulse of $N_{s c p}$ samples in $\zeta_{N}(m)$ with the time-delayed instantaneous channel power delay profile defined as ${ }^{4}$

$$
\gamma\left(m ; \boldsymbol{\Theta}_{\sim \nu}\right) \doteq \sum_{k=0}^{L_{h}-1}\left|h_{k}\right|^{2} \operatorname{sinc}(m-k-\tau) .
$$

In view of this effect, the basic waveform being repeated along the $\pm N$-th diagonals of the multipath-affected $\mathbf{M}(\boldsymbol{\Theta})$ matrix is now given by,

$$
\begin{aligned}
\zeta_{N}\left(m ; \boldsymbol{\Theta}_{\sim \nu}\right) & =\operatorname{rect}_{N_{s c p}}(m) * \gamma\left(m ; \boldsymbol{\Theta}_{\sim \nu}\right) \\
& =\sum_{k=0}^{L_{h}-1}\left|h_{k}\right|^{2} \operatorname{rect}_{N_{s c p}}(m-k-\tau)
\end{aligned}
$$

which is a superposition of time-shifted and scaled rectangular pulses (or interpolated rectangular pulses for fractional $\tau$ ), with the scaling factors being the squared absolute value of each channel tap gain. In matrix notation, (80) can be expressed in a more convenient and compact form as follows:

$$
\boldsymbol{\zeta}_{N}\left(\Theta_{\sim \nu}\right)=\Pi(\tau) \boldsymbol{\gamma}
$$

where $\zeta_{N}\left(\boldsymbol{\Theta}_{\sim \nu}\right) \doteq\left[\zeta_{N}\left(0 ; \boldsymbol{\Theta}_{\sim \nu}\right), \ldots, \zeta_{N}\left(N_{s s}-1 ; \boldsymbol{\Theta}_{\sim \nu}\right)\right]^{T}$ is a $\left(N_{s s} \times 1\right)$ vector that contains the samples of the basic waveform $\zeta_{N}\left(m ; \boldsymbol{\Theta}_{\sim \nu}\right), \boldsymbol{\Pi}(\tau)$ is a $\left(N_{s s} \times L_{h}\right)$ matrix whose columns are one-sample time-shifted replicas of a rectangular pulse delayed by $\tau$, and $\gamma \doteq\left[\left|h_{0}\right|^{2}, \ldots,\left|h_{L_{h}-1}\right|^{2}\right]^{T}$ contains the $L_{h}$ coefficients of the instantaneous power delay profile $\gamma\left(m ; \boldsymbol{\Theta}_{\sim \nu}\right)$ in (78).

The problem of time-delay and frequency estimation is now coupled with that of channel estimation, which is required in order to determine the shape of the waveform $\zeta_{N}\left(m ; \boldsymbol{\Theta}_{\sim \nu}\right)$ where the time-delay and frequency estimates are embedded. However, just the squared values of the channel tap gains are required, as indicated in (80). Thus, taking into account the equivalent matrix notation in (81), the low-SNR UML cost function in (72) can be recast as follows for the case of multipath channels,

$$
\begin{aligned}
L_{\mathrm{UML}}^{\prime \prime}(\mathbf{r} \mid \boldsymbol{\Theta})=2 \operatorname{Re}\left[e^{j 2 \pi \nu} \boldsymbol{\zeta}_{N}^{H}\left(\boldsymbol{\Theta}_{\sim \nu}\right) \tilde{\mathbf{r}}_{r}(N)\right] \\
+L \boldsymbol{\zeta}_{N}^{H}\left(\boldsymbol{\Theta}_{\sim \nu}\right) \zeta_{N}\left(\boldsymbol{\Theta}_{\sim \nu}\right)
\end{aligned}
$$

where $\tilde{\mathbf{r}}_{r}(N) \doteq\left[\tilde{R}_{r}(0 ; N), \ldots, \tilde{R}_{r}\left(N_{s s-1} ; N\right)\right]^{T}$. As in the single-path case, the frequency estimation is decoupled from the rest of parameters and it can be compressed back into the cost function, resulting in

$$
\begin{aligned}
L_{\mathrm{UML}}^{\prime \prime}\left(\mathbf{r} \mid \Theta_{\sim \nu}\right)=\mid 2 \boldsymbol{\zeta}_{N}^{H}\left(\boldsymbol{\Theta}_{\sim \nu}\right) & \tilde{\mathbf{r}}_{r}(N) \\
& +L \boldsymbol{\zeta}_{N}^{H}\left(\boldsymbol{\Theta}_{\sim \nu}\right) \boldsymbol{\zeta}_{N}\left(\boldsymbol{\Theta}_{\sim \nu}\right) \mid .
\end{aligned}
$$

At this point, the problem becomes that of estimating an unknown time-delay $\tau$ embedded in an unknown waveform $\zeta_{N}\left(\Theta_{\sim \nu}\right)$, a coupled problem because the shape of the wave-

\footnotetext{
${ }^{4}$ The notation $\Theta_{\sim \nu}$ denotes the set of nuisance parameters in $\Theta$ excluding $\nu$
}

form itself depends on the time-delay, too. However, we can solve this problem following a two-step approach: first we can determine the unknown waveform coefficients $\gamma$ for a fixed $\tau$ and then compress them back into the cost function; second, we can proceed with the maximization of (83) as a function of $\tau$ only. Let us begin with the estimation of the unknown waveform. Since $L_{\mathrm{UML}}^{\prime \prime}\left(\mathbf{r} \mid \Theta_{\sim \nu}\right)>0$, we can apply the square function $g(x)=x^{2}$ to it, which is a monotonic function for $x>0$ and thus it preserves the maximum points of $L_{\mathrm{UML}}^{\prime \prime}\left(\mathbf{r} \mid \Theta_{\sim \nu}\right)$. By doing so, and taking into account the relationship in (81), we can easily differentiate with respect to $\boldsymbol{\gamma}$ and obtain,

$$
\hat{\boldsymbol{\gamma}}(\tau)=C_{\gamma}\left[\boldsymbol{\Pi}^{H}(\tau) \boldsymbol{\Pi}(\tau)\right]^{-1} \boldsymbol{\Pi}^{H}(\tau) \tilde{\mathbf{r}}_{r}(n)
$$

which is a function of $\tau$ and $C_{\gamma}$, a positive scaling factor irrelevant for time-delay estimation. Compressing this estimate into (83), and after some manipulations, we get the compact expression

$$
L_{\mathrm{UML}}^{\prime \prime}(\mathbf{r} \mid \tau) \propto\left|\mathbf{P}_{\Pi(\tau)} \tilde{\mathbf{r}}_{r}(N)\right| .
$$

where $\mathbf{P}_{\boldsymbol{\Pi}(\tau)} \doteq \boldsymbol{\Pi}(\tau)\left[\boldsymbol{\Pi}(\tau)^{H} \boldsymbol{\Pi}(\tau)\right]^{-1} \boldsymbol{\Pi}(\tau)^{H}$ is the projection matrix onto the subspace spanned by $\Pi(\tau)$ in (81). Then, the low-SNR UML time-delay and frequency estimator for multipath channels becomes,

$$
\begin{aligned}
& \hat{\tau}_{\mathrm{UML}}=\arg \max _{\tau}\left\{\left|\mathbf{P}_{\boldsymbol{\Pi}(\tau)} \tilde{\mathbf{r}}_{r}(N)\right|\right\} \\
& \hat{\nu}_{\mathrm{UML}}=\frac{1}{2 \pi} \arg \left\{\hat{\boldsymbol{\gamma}}^{H}\left(\hat{\tau}_{\mathrm{UML}}\right) \boldsymbol{\Pi}^{H}\left(\hat{\tau}_{\mathrm{UML}}\right) \tilde{\mathbf{r}}_{r}\right\} .
\end{aligned}
$$

\section{Simulation Results}

Computer simulations have been carried out to assess the performance of the techniques derived in Sections IV and V for CP-OFDM signals in both single-path (i.e., AWGN) and multipath channels. We consider herein a default CP-OFDM signal with $N=64$ subcarriers and a CP of $N_{s c p}=10$ samples, similar to the lowest bandwidth LTE signal with non-extended CP ([29], Section E.5.1). A comparison is established with the technique proposed in [9], which is the most widely adopted method for blind synchronization of CP-OFDM signals.

Experiment 1-Performance in Single-Path Channels: This is the reference working scenario in which traditional synchronizers for CP-OFDM signals were typically derived, such as the well-known Beek's ML estimator [9]. As shown in Fig. 5, this estimator coincides with the low-SNR UML estimator proposed in (73)-(74) for the low-SNR regime, whereas it coincides with the CML estimator in (63)-(66) for the high-SNR regime. These results are consistent with the discussion at the end of Section V-A, and they suggest that when operating under either of these two regimes, complexity can be reduced by substituting Beek's estimator by either the proposed low-SNR UML or the CML estimator, which do not require the actual SNR to be estimated. Note that the performance of all these estimators tends to saturate at high-SNR due to the finite observation interval for which the ML cost function is evaluated. In [9], this floor effect is not observed (although it is actually present but at an even higher SNR) because of the very large observation interval used therein, where each Montecarlo simulation is averaged over a total of $L=125,000$ OFDM 

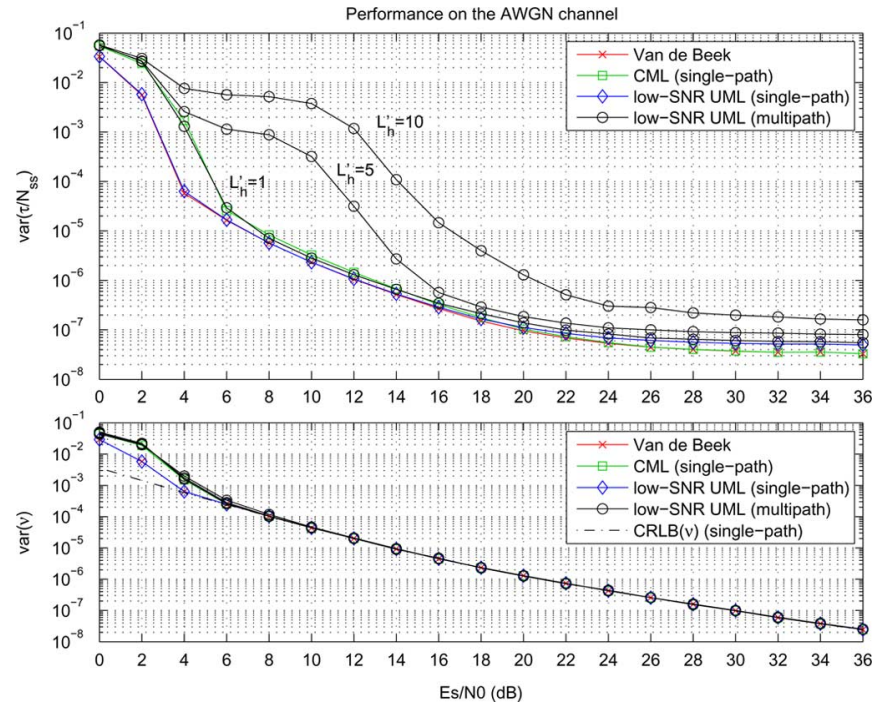

Fig. 5. Variance of time-delay and frequency estimates for a CP-OFDM signal with $N_{s s}=74\left(N=64\right.$ subcarriers, $\left.N_{s c p}=10\right)$ and $L=2000$ OFDM symbols transmitted through a single-path (i.e., AWGN) channel.

symbols with $N=256$ subcarriers, compared to $L=2,000$ and $N=64$ considered herein. For the low-SNR UML estimator, and because of its low-SNR assumption, an additional penalty is incurred when operating in the high-SNR regime, where an increased floor effect is observed in Fig. 5. Regarding the low-SNR UML estimator for multipath channels, its performance degrades due to the increased number of unknowns (i.e., the time-delay $\tau$ and the $L_{h}^{\prime}$ taps $^{5}$ from the power delay profile, according to (84)) that must be handled. In this experiment, even though the channel is single-path, we are assuming $L_{h}^{\prime}=\{1,5,10\}$ in order to assess the degradation incurred when increasing $L_{h}^{\prime}$. For the carrier frequency estimation, the Cramér-Rao bound given by $\operatorname{CRB}(\nu)=\frac{2 \mathrm{SNR}+1}{8 \pi^{2} L\left(N_{s c p}+1\right) \mathrm{SNR}^{2}}$ has been included as a reference benchmark [30], which is attained by all single-path estimators in Fig. 5 .

Experiment 2-Performance in Multipath Channels With Short Delay Spread: In this experiment we adopt the 3GPP Rural Area channel model (RAx) [31], which for a sampling time of $0.1 \mu \mathrm{s}$ leads to an effective channel length of $L_{h}=6$ taps 6 . For the proposed low-SNR UML estimator in (86), we consider $L_{h}^{\prime}=\{2,4,6,8\}$ taps. The results in the upper plot of Fig. 6 show how traditional time-delay estimators for CP-OFDM exhibit a performance floor beyond $E_{s} / N_{0}=12$ $\mathrm{dB}$. The proposed estimator in (86) also reaches a floor but for much higher $E_{s} / N_{0}$ values. In the meantime, it is able to reduce the time-delay variance by two orders of magnitude compared to traditional estimators due to its joint time-delay and channel estimation. It is interesting to observe the different performance as a function of $L_{h}^{\prime}$, showing how the increase in the number of channel taps produces a penalty in the time-delay

${ }^{5}$ Note that $L_{h}^{\prime}$ does not need to coincide with the actual number of channel taps, $L_{h}$. In practice, $L_{h}^{\prime}$ is typically set to capture the most significant taps while keeping a reduced number of unknowns to be estimated. Thus, it often happens that $L_{h}^{\prime} \leq L_{h}$.

${ }^{6}$ In experiments where statistical channel models are involved, the time-delay estimation problem may become ill-conditioned when the first tap of the actual channel realization is very small. To avoid this problem, only those channel realizations whose first path is at least $40 \%$ of the total channel energy have been selected for simulation purposes.
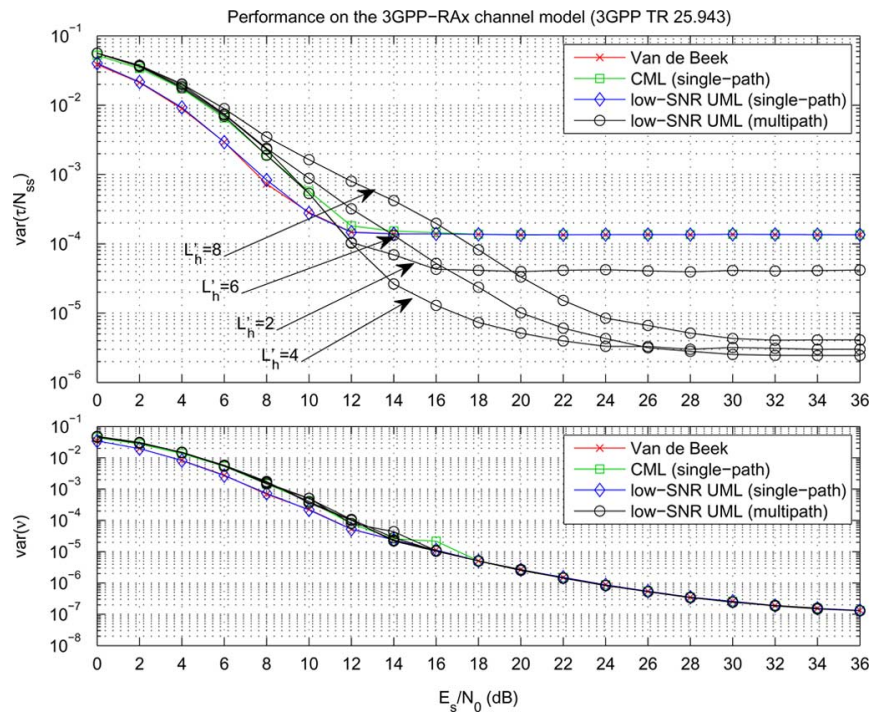

Fig. 6. Variance of time-delay and frequency estimates for a CP-OFDM signal with $N_{s s}=74\left(N=64\right.$ subcarriers, $\left.N_{s c p}=10\right)$ and $L=2000$ OFDM symbols transmitted through the 3GPP Rural Area channel model (RAx) [31].

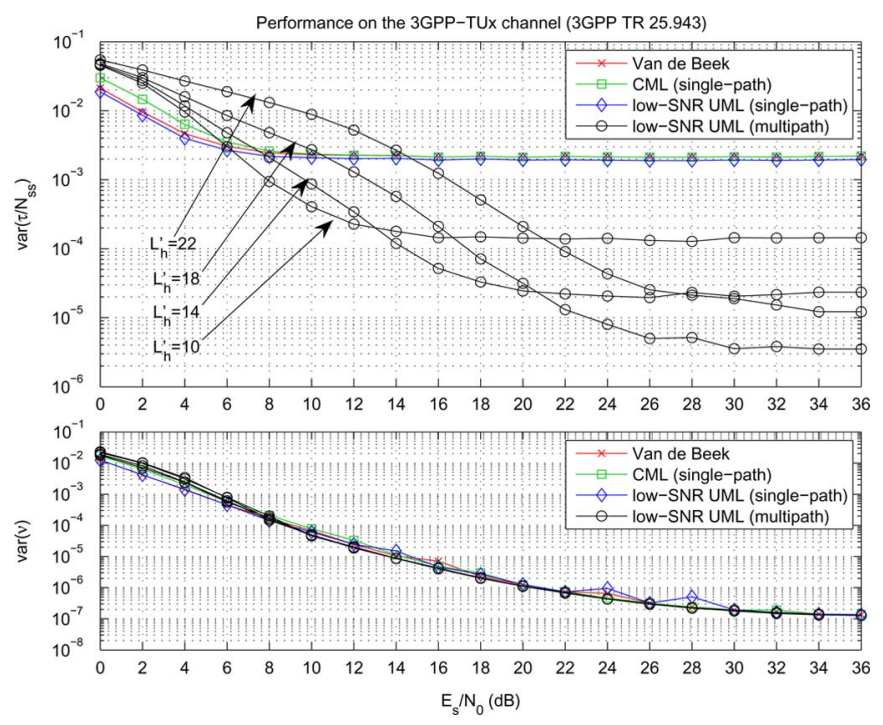

Fig. 7. Variance of time-delay and frequency estimates for a CP-OFDM signal with $N_{s s}=74\left(N=52\right.$ subcarriers, $\left.N_{s c p}=22\right)$ and $L=2000$ OFDM symbols transmitted through the 3GPP Typical Urban channel model (TUx) [31].

but not the frequency estimation performance, which appears to be rather insensitive to the presence of multipath.

Experiment 3-Performance in Multipath Channels With Long Delay Spread: In this case we adopt the 3GPP Typical Urban channel (TUx) [31], which for the same sampling rate as the previous experiment leads to an effective channel length of $L_{h}=22$ taps. Such a long delay spread introduces a significant degradation in the overall time-delay estimation performance of traditional methods, which do not take into account the presence of the channel. In contrast, the proposed method in (86) is able to provide an improvement of almost three orders of magnitude compared to traditional methods, leading to similar results (e.g., for $L_{h}^{\prime}=18$ ) to the ones obtained in Fig. 6 for a shorter delay spread. This result confirms the robustness of the proposed method and its ability to combat the presence of multipath. 


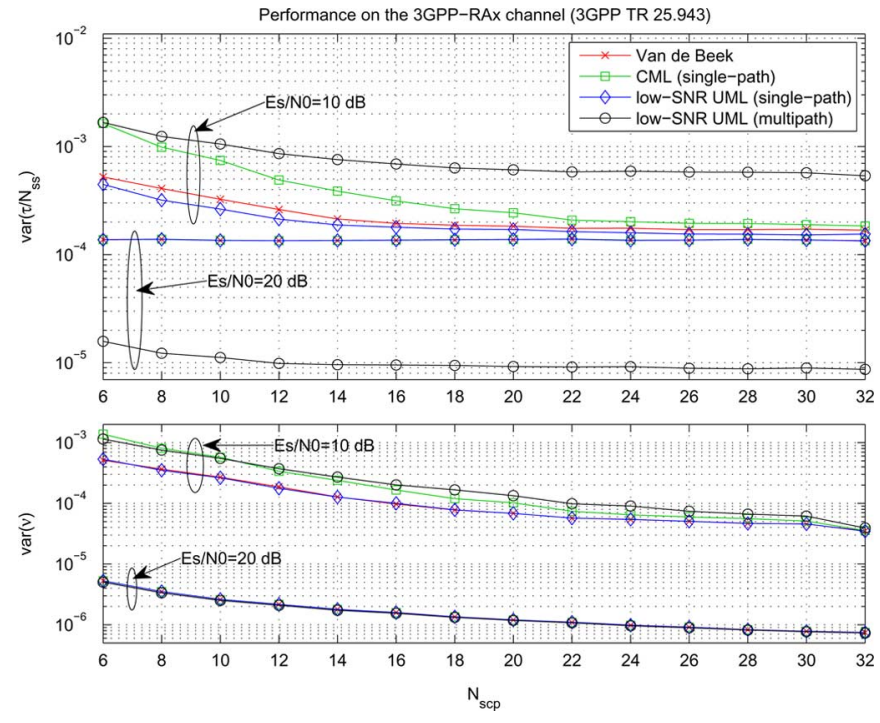

Fig. 8. Variance of time-delay and frequency estimates for a CP-OFDM signal with $N_{s s}=74, L_{h}^{\prime}=6$ estimated taps and $L=2000$ OFDM symbols transmitted through the 3GPP-RAx channel as a function of the cyclic prefix length $N_{s c p}$.

Experiment 4-Performance as a Function of $N_{\text {scp }}$ : This experiment evaluates the impact of the cyclic prefix length onto the overall synchronization performance. The 3GPP RAx channel model has been selected here, for which a minimum cyclic prefix of $N_{s c p}=6$ samples has been set (i.e., $N_{s c p}=L_{h}$ ). As can be observed in Fig. 8 for $E_{s} / N_{0}=\{10,20\} \mathrm{dB}$, the performance improvement due to the cyclic prefix length is only visible at low $E_{s} / N_{0}$ values. This is in line with the results in [9], which suggest that beyond some $E_{s} / N_{0}$ threshold, there is not a significant change in time-delay estimation performance when varying the cyclic prefix, provided that it is large enough to encompass the channel delay spread. This observation, however, does not apply to the frequency estimates, which are found to monotically improve as the cyclic prefix increases.

\section{CONCLUSION}

A unified framework has been presented in this paper by introducing a novel matrix signal model for the general class of flexible FBMC signals. This framework encompasses many possible signal designs in terms of pulse shaping filter, symbol duration and subcarrier spacing, in contrast to most of the existing $\mathrm{MC}$ signaling formats where these parameters are typically constrained. Moreover, the proposed matrix signal model has allowed us to easily derive both the CML and the low-SNR UML time-delay and frequency estimation of an arbitrary FBMC signal propagating through an arbitrary multipath channel. Based on these results, the special case of critically sampled CP-OFDM signals has been addressed, where a simple and novel estimator has been proposed for blind synchronization in multipath channels.

\section{APPENDIX A}

\section{VERIFICATION OF THE ORTHOGONALITY CONDITION}

In single-carrier communication signals, the adoption of square-root Nyquist pulses guarantees that $\mathbf{A}^{H}(\boldsymbol{\Theta}) \mathbf{A}(\boldsymbol{\Theta})=$ $E_{g} \mathbf{I}$, where $E_{g}$ is the energy of the pulse. The intuitive interpretation is that, after being decimated by a factor $N_{s s}$ and having the receiver synchronized to the transmitter, the matched filter output for $\mathbf{A}(\boldsymbol{\Theta})$ allows an inter-symbol interference (ISI)-free recovery of the transmitted symbols. For multicarrier signals, this result must be extended to incorporate the additional constraint of avoiding inter-carrier interference (ICI). In these circumstances, the following lemma applies:

Lemma 2: For the FBMC shaping matrix defined in (12), its inner product can be expressed as,

$\mathbf{A}^{H}(\boldsymbol{\Theta}) \mathbf{A}(\boldsymbol{\Theta})$

$$
=\left[\begin{array}{cccc}
\mathbf{D}_{-K,-K} & \mathbf{D}_{-K,-K+1} & & \mathbf{D}_{-K, K} \\
\mathbf{D}_{-K+1,-K} & \mathbf{D}_{-K+1,-K+1} & \mathbf{D}_{-K+1, K} \\
\vdots & \vdots & \cdots & \vdots \\
\mathbf{D}_{K,-K} & \mathbf{D}_{K,-K+1} & & \mathbf{D}_{K, K}
\end{array}\right]
$$

where $\mathbf{D}_{l_{1}, l_{2}} \doteq \tilde{\mathbf{F}}_{\tau} \operatorname{diag}\left(\mathbf{g}_{l_{1}}(\boldsymbol{\Theta}) \odot \mathbf{g}_{l_{2}}^{*}(\boldsymbol{\Theta})\right) \tilde{\mathbf{F}}_{\tau}^{H}$, with $\left\{l_{1}, l_{2}\right\}=$ $-K, \ldots, K$, is an $(N \times N)$ submatrix.

Proof: The first step is to substitute $\mathbf{A}(\boldsymbol{\Theta})$ with its definition in (12):

$$
\begin{array}{r}
\mathbf{A}^{H}(\boldsymbol{\Theta}) \mathbf{A}(\boldsymbol{\Theta}) \\
=\left(\mathbf{G}^{H}(\boldsymbol{\Theta}) \otimes \tilde{\mathbf{F}}_{\tau}\right) \mathbf{J}_{1} \mathbf{J}_{1}^{T}\left(\mathbf{G}(\boldsymbol{\Theta}) \otimes \tilde{\mathbf{F}}_{\tau}^{H}\right)
\end{array}
$$

where the outer-product of the selection matrix $\mathbf{J}_{1}=\mathbf{I}_{Q} \diamond \mathbf{I}_{Q}$ can be rewritten by defining the $(Q \times 1)$ vector $\mathbf{v}_{q} \doteq\left[\mathbf{I}_{Q}\right]_{:, q}$ as the $q$-th column of the $(Q \times Q)$ identity matrix $\mathbf{I}_{Q}$. As a result, we have that

$$
\begin{aligned}
\mathbf{J}_{1} \mathbf{J}_{1}^{T}= & {\left[\mathbf{v}_{-M} \otimes \mathbf{v}_{-M}, \ldots, \mathbf{v}_{M} \otimes \mathbf{v}_{M}\right] } \\
& \times\left[\mathbf{v}_{-M} \otimes \mathbf{v}_{-M}, \ldots, \mathbf{v}_{M} \otimes \mathbf{v}_{M}\right]^{T} \\
= & \sum_{q=-M}^{M}\left(\mathbf{v}_{\mathbf{q}} \mathbf{v}_{q}^{T}\right) \otimes\left(\mathbf{v}_{\mathbf{q}} \mathbf{v}_{q}^{T}\right)=\operatorname{diag}\left(\operatorname{vec}\left(\mathbf{I}_{Q}\right)\right)
\end{aligned}
$$

From (91), it can be seen that the outer-product $\mathbf{J}_{\mathbf{1}} \mathbf{J}_{\mathbf{1}}{ }^{T}$ is indeed a $\left(Q^{2} \times Q^{2}\right)$ diagonal matrix. In particular, its diagonal elements turn out to be all zero except for the ones in the $k(Q+1)+1$ entries, with $k=0,1, \ldots, Q-1$. As such, the matrix $\mathbf{J}_{1} \mathbf{J}_{1}^{T}$ can be understood as a selection matrix that removes the redundancy of the Kronecker product matrices on its left or right hand side in (89). For instance, for the right hand side multiplication in (89), it is easy to show that,

$$
\begin{aligned}
& \mathbf{J}_{1} \mathbf{J}_{1}^{T}\left(\mathbf{G}(\boldsymbol{\Theta}) \otimes \tilde{\mathbf{F}}_{\tau}^{H}\right) \\
& =\left[\operatorname{diag}\left([\mathbf{G}(\boldsymbol{\Theta})]_{:,-K}\right) \tilde{\mathbf{F}}_{\tau}^{H}, \ldots, \operatorname{diag}\left([\mathbf{G}(\boldsymbol{\Theta})]_{:, K}\right) \tilde{\mathbf{F}}_{\tau}^{H}\right] .
\end{aligned}
$$

Similarly for the left hand side product in (89),

$$
\begin{aligned}
& \left(\mathbf{G}^{H}(\boldsymbol{\Theta}) \otimes \tilde{\mathbf{F}}_{\tau}\right) \mathbf{J}_{1} \mathbf{J}_{1}^{T}
\end{aligned}
$$

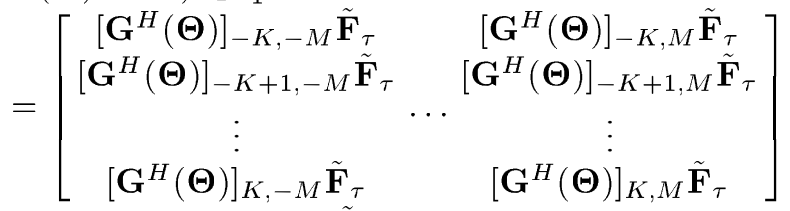

$$
\begin{aligned}
& \times \operatorname{diag}\left(\operatorname{vec}\left(\mathbf{I}_{Q}\right)\right)\left[\begin{array}{c}
\tilde{\mathbf{F}}_{\tau} \operatorname{diag}\left(\left[\mathbf{G}^{*}(\boldsymbol{\Theta})\right]_{:,-K}\right) \\
\tilde{\mathbf{F}}_{\tau} \operatorname{diag}\left(\left[\mathbf{G}^{*}(\boldsymbol{\Theta})\right]_{:,-K+1}\right) \\
\vdots \\
\tilde{\mathbf{F}}_{\tau} \operatorname{diag}\left(\left[\mathbf{G}^{*}(\boldsymbol{\Theta})\right]_{:, K}\right)
\end{array}\right] .
\end{aligned}
$$

Finally, using (92), (94) and the idempotency of $\mathbf{J}_{1} \mathbf{J}_{1}^{T}$, we can rewrite (89) as indicated in (88). 
Based on Lemma 2, ISI-free and ICI-free FBMC signals are possible provided that $\mathbf{A}^{H}(\boldsymbol{\Theta}) \mathbf{A}(\mathbf{\Theta})=E_{g} \mathbf{I}_{L N}$, which implies that $\mathbf{D}_{l_{1}, l_{2}}=\mathbf{0}_{N}$ for $l_{1} \neq l_{2}$ and $\mathbf{D}_{l_{1}, l_{1}}=E_{g} \mathbf{I}_{N}$.

\section{REFERENCES}

[1] T. Hwang, C. Yang, G. Wu, S. Li, and G. Y. Li, "OFDM and its wireless applications: A survey," IEEE Trans. Veh. Technol., vol. 58, no. 4, pp. 1673-1694, May 2009.

[2] W. Yu, G. Ginis, and J. M. Cioffi, "Distributed multiuser power control for digital subscriber lines," vol. 20, no. 5, pp. 1105-1115, Jun. 2002.

[3] G. Song and Y. Li, "Utility-based resource allocation and scheduling in OFDM-based wireless broadband networks," IEEE Commun. Mag., vol. 6, no. 6, pp. 127-134, Dec. 2005.

[4] B. Farhang-Boroujeny, "OFDM versus filter bank multicarrier," IEEE Signal Process. Mag., vol. 28, no. 3, pp. 92-112, May 2011.

[5] Z. Kollár and P. Horváth, "Physical layer considerations for cognitive radio: Synchronization point of view," in Proc. IEEE Veh. Technol. Conf. (VTC), May 2011, pp. 1-5.

[6] E. Gutiérrez, J. A. López-Salcedo, and G. Seco-Granados, "Unified framework for flexible multi-carrier communication systems," presented at the 8th Int. Workshop on Multi-Carrier Syst. Solutions, Herrsching, Germany, May 2011.

[7] T. Fusco, A. Petrella, and M. Tanda, "Data-aided symbol timing and CFO synchronization for filter bank multicarrier systems," IEEE Trans. Wireless Commun., vol. 8, no. 5, pp. 2705-2715, May 2009.

[8] M. Sandell, J.-J. van de Beek, and P. O. Börjesson, "Timing and frequency synchronization in OFDM systems using the cyclic prefix," in Proc. Int. Symp. Synchroniz., Essen, Germany, Dec. 1995, pp. 16-19.

[9] J.-J. van de Beek, M. Sandell, and P. O. Börjesson, "ML estimation of time and frequency offset in OFDM systems," IEEE Trans. Signal Process., vol. 45, no. 7, pp. 1800-1805, Jul. 1997.

[10] S. Wei, D. L. Goeckel, and P. A. Kelly, "Convergence of the complex envelope of bandlimited OFDM signals," IEEE Trans. Inf. Theory, vol. 56, no. 10, pp. 4893-4904, Oct. 2010.

[11] H. Bölcskei, "Blind estimation of symbol timing and carrier frequency offset in wireless OFDM systems," IEEE Trans. Commun., vol. 49, no. 6, pp. 988-999, Jun. 2001.

[12] F. Gini and G. B. Giannakis, "Frequency offset and symbol timing recovery in flat-fading channels: A cyclostationary approach," IEEE Trans. Commun., vol. 46, pp. 400-411, Mar. 1998.

[13] B. Park, H. Cheon, E. Ko, C. Kang, and D. Hong, "A blind OFDM synchronization algorithm based on cyclic correlation," IEEE Signal Process. Lett., vol. 11, no. 2, pp. 83-85, Feb. 2004.

[14] A. Laourine, A. Stephanne, and S. Affes, "Blind sampling clock offset estimation in OFDM systems based on second order statistics," in Proc. 40th Asilomar Conf. Signals, Syst. Comput., Oct. 29-Nov. 1 2006, pp. 1782-1785.

[15] V. Le Nir, T. van Waterschoot, M. Moonen, and J. Duplicy, "Blind CP-OFDM and ZP-OFDM parameter estimation in frequency selective channels," EURASIP J. Wireless Commun. Netw., pp. 1-10, 2009.

[16] G. Vázquez and J. Riba, "Non-data-aided digital synchronization," in Signal Processing Advances in Wireless and Mobile Communications. Englewood Cliffs, NJ: Prentice-Hall, 2000, vol. 2, ch. 9.

[17] S. Liu and G. Trenkler, "Hadamard, Katri-Rao, Kronecker and other matrix products," Int. J. Inf. Syst. Sci., vol. 4, no. 1, pp. 160-177, 2008.

[18] V. Tripathi, A. Mantravadi, and V. V. Veeravalli, "Channel acquisition for wideband CDMA signals," IEEE J. Sel. Areas Commun., vol. 18, no. 8, pp. 1483-1494, Aug. 2000.

[19] M. D. Larsen, G. Seco-Granados, and A. L. Swindlehurst, "Pilot optimization for time-delay and channel estimation in OFDM systems," in Proc. IEEE Int. Conf. on Acoust., Speech Signal Process. (ICASSP), 2011, pp. 3564-3567.

[20] R. Mo, Y. H. Chew, T. T. Tjhung, and C. C. Ko, "A joint blind timing and frequency offset estimator for OFDM systems over frequency selective fading channels," IEEE Trans. Wireless Commun., vol. 5, no. 9, pp. 2604-2614, Sep. 2006.

[21] U. Mengali and A. N. D'Andrea, Synchronization Techniques for Digital Receivers. New York: Plenum, 1997.

[22] P. Chevillat, D. Maiwald, and G. Ungerboeck, "Rapid training of a voiceband data-modem receiver employing an equalizer with fractional-T spaced coefficients," IEEE Trans. Commun., vol. 35, no. 9, pp. 869-876, Sep. 1987.

[23] S. H. Müller-Weinfurtner, "On the optimality of metrics for coarse frame synchronization in OFDM: A comparison," in Proc. IEEE Int. Symp. on Pers., Indoor Mobile Radio Commun. (PIMRC), 1998, pp. 533-537.
[24] T. Keller and L. Hanzo, "Orthogonal frequency division multiplex synchronisation techniques for wireless local area networks," in Proc. IEEE Int. Symp. on Pers., Indoor Mobile Radio Commun. (PIMRC), 1996, pp. 963-967.

[25] R. Negi and J. M. Cioffi, "Blind OFDM symbol synchronization in ISI channels," IEEE Trans. Commun., vol. 50, no. 9, pp. 1525-1534, Sep. 2002.

[26] J.-J. Van de Beek, P. O. Börjesson, M.-L. Boucheret, D. Landström, J. M. Arenas, P. Ödling, C. Ostberg, M. Wahlqvist, and S. K. Wilson, "A time and frequency synchronization scheme for multiuser OFDM," IEEE J. Sel. Areas Commun., vol. 17, no. 11, pp. 1900-1914, Nov. 1999.

[27] B. Yang, K. B. Letaief, R. S. Cheng, and Z. Cao, "Timing recovery for OFDM transmissions," IEEE J. Sel. Areas Commun., vol. 18, no. 11, pp. 2278-2291, Nov. 2000.

[28] S. Ma, X. Pan, G.-H. Yang, and T.-S. Ng, "Blind symbol synchronization based on cyclic prefix for OFDM systems," IEEE Trans. Veh. Technol., vol. 58, no. 4, pp. 1746-1751, May 2009.

[29] 3GPP, "Evolved Universal Terrestrial Radio Access (E-UTRA); Base Station (BS) Radio Transmission and Reception," TS 136.104 LTE, Jan. 2011, V10.1.0.

[30] W.-L. Chin, "ML estimation of timing and frequency offsets using distinctive correlation characteristics of OFDM signals over dispersive fading channels," IEEE Trans. Veh. Commun., vol. 60, no. 2, pp. 444-456, Feb. 2011

[31] 3GPP, "Universal Mobile Telecommunications System (UMTS); Deployment Aspects," TR 25 943, May 2011, V10.0.0.

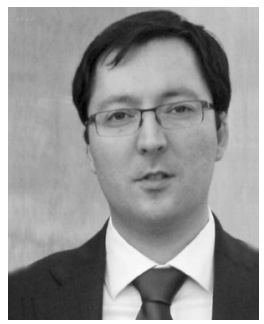

José A. López-Salcedo (S'98-M'03) received the M.S. and Ph.D. degrees in telecommunication engineering from the Universitat Politècnica de Catalunya (UPC), Barcelona, Spain, in 2001 and 2007, respectively.

From 2002 to 2006, he was a Research Assistant at UPC, where he was involved in more than 10 research projects related to synchronization techniques for digital receivers, satellite communications, and iterative decoding algorithms for MIMO wireless systems. Since 2006, he has been an Assistant Professor with the Department of Telecommunications and Systems Engineering, Universitat Autònoma de Barcelona (UAB), where he has been involved in several research projects funded by the European Space Agency (ESA), related to the design of high-sensitivity receivers for GPS and Galileo, as well as the design of robust carrier tracking techniques. So far, he has been involved in more than 24 national and international research projects, and he has been principal investigator of more than 8 of them. Since May 2011, he has been coordinator of the Telecommunications Engineering degree at the UAB Engineering School. In summer 2011, he was a visiting scholar at the Coordinated Science Laboratory (CSL), University of Illinois at Urbana-Champaign. During 2010-2012, he also had several visiting appointments with the University of California at Irvine, in the framework of the California-Catalonia Engineering Innovation Program funded by the Balsells Fellowship. His research interests include statistical signal processing, detection and estimation theory, synchronization techniques for digital receivers and applications to wireless communications and navigation.

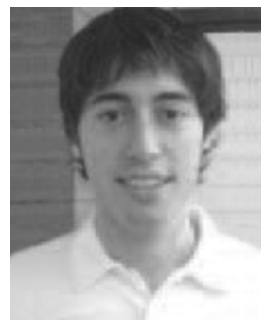

Esteban Gutiérrez received the M.S. degree in telecommunications engineering and a master's degree in aerospace science and technology from the Universitat Politècnica de Catalunya (UPC), Barcelona, Spain, in 2006 and 2010, respectively.

In 2007, he joined the Aerospace Research and Technology Centre (Ascamm Foundation) where he works as a researcher in the Navigation and Communications area. $\mathrm{He}$ is currently pursuing the Ph.D. degree with the Signal Processing for Communications and Navigation Research Group, Universitat Autònoma de Barcelona (UAB). His research interests are digital signal processing and wireless communications with emphasis on multicarrier systems. 


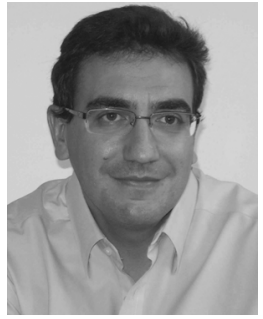

Gonzalo Seco-Granados (S'97-M'02-SM'08) received the $\mathrm{Ph} . \mathrm{D}$. degree in telecommunication engineering from the Universitat Politècnica de Catalunya (UPC), Barcelona, Spain, in 2000, and the M.B.A. degree from IESE-University of Navarra, Barcelona, in 2002.

During 2002-2005, he was a member of the technical staff within the RF Payload Division, European Space Research and Technology Center (ESTEC), European Space Agency, Noordwijk, The Netherlands, where he was involved in the Galileo project. He led the activities concerning navigation receivers and indoor positioning for GPS and Galileo. Since 2006, he has been an Associate Professor with the Department of Telecommunications and Systems Engineering, Universitat Autònoma de Barcelona, Barcelona. From March 2007 to April 2011, he was coordinator of the Telecommunications Engineering degree and, since May 2011, he has been vice director of the UAB Engineering School. He has been principal investigator of more than 12 national and international research projects, and acts often as an advisor of the European Commission in topics related to communications and navigation. He has had several visiting appointments at Brigham Young University, Provo, UT, and the University of California at Irvine. He has published more than 20 journal papers and more than 80 conference contributions. He holds two patents under exploitation. His research interests include signal processing for wireless communications and navigation, estimation theory, synchronization, location-based communications and optimization.

Dr. Seco-Granados was appointed Director of one of the six Chairs of Technology and Knowledge Transfer "UAB Research Park-Santander" in March 2008 .

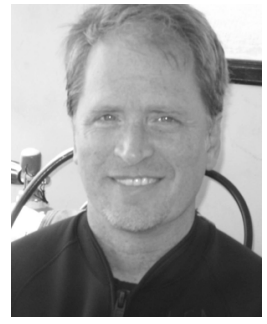

A. Lee Swindlehurst (S'83-M'84-SM'99-F'04) received the B.S. (summa cum laude) and M.S. degrees in electrical engineering from Brigham Young University, Provo, UT, in 1985 and 1986, respectively, and the Ph.D. degree in electrical engineering from Stanford University, Stanford, CA, in 1991.

From 1986 to 1990, he was with ESL, Inc., Sunnyvale, CA, where he was involved in the design of algorithms and architectures for several radar and sonar signal processing systems. He was on the faculty of the Department of Electrical and Computer Engineering, Brigham Young University, from 1990 to 2007, where he was a Full Professor and served as Department Chair from 2003 to 2006. During 1996-1997, he held a joint appointment as a visiting scholar at both Uppsala University, Uppsala, Sweden, and at the Royal Institute of Technology, Stockholm, Sweden. From 2006-2007, he was on leave working as Vice President of Research for ArrayComm LLC, San Jose, CA. He is currently a Professor of Electrical Engineering and Computer Science, University of California at Irvine. His research interests include sensor array signal processing for radar and wireless communications, detection and estimation theory, and system identification, and he has more than 220 publications in these areas.

Dr. Swindlehurst is a past Secretary of the IEEE Signal Processing Society, past Editor-in-Chief of the IEEE JOURNAL OF SELECTED TOPICS IN SIGNAL PROCESSING, and past member of the Editorial Boards for the EURASIP Journal on Wireless Communications and Networking, IEEE Signal Processing Magazine, and the IEEE TRANSACTIONS ON SIGNAL PROCESSING. He is a recipient of several paper awards: the 2000 IEEE W. R. G. Baker Prize Paper Award, the 2006 and 2010 IEEE Signal Processing Society Best Paper Award, the 2006 IEEE Communications Society Stephen O. Rice Prize in the Field of Communication Theory, and is coauthor of a paper that received the IEEE Signal Processing Society Young Author Best Paper Award in 2001. 